\title{
Optimum efficiency control of a wind turbine with unknown desired trajectory and actuator faults
}

Hamed Habibi, Hamed Rahimi Nohooji, and lan Howard

Citation: Journal of Renewable and Sustainable Energy 9, 063305 (2017); doi: 10.1063/1.5003380

View online: https://doi.org/10.1063/1.5003380

View Table of Contents: http://aip.scitation.org/toc/rse/9/6

Published by the American Institute of Physics

\section{Articles you may be interested in}

Comparison of different dynamic models for floating wind turbines

Journal of Renewable and Sustainable Energy 9, 063304 (2017); 10.1063/1.5002750

An improved control strategy to the frequency regulation of DFIG based wind turbine Journal of Renewable and Sustainable Energy 9, 063303 (2017); 10.1063/1.4990108

A probabilistic method for cost minimization in a day-ahead electricity market considering wind power uncertainties

Journal of Renewable and Sustainable Energy 9, 063301 (2017); 10.1063/1.4987037

Effects of second-order wave forces and aerodynamic forces on dynamic responses of a TLP-type floating offshore wind turbine considering the set-down motion

Journal of Renewable and Sustainable Energy 9, 063302 (2017); 10.1063/1.5007893

Experimental study of the accumulative deformation effect on wide-shallow composite bucket foundation for offshore wind turbines

Journal of Renewable and Sustainable Energy 9, 063306 (2017); 10.1063/1.5017464

Dynamic response and mooring optimization of spar-type substructure under combined action of wind, wave, and current

Journal of Renewable and Sustainable Energy 9, 063307 (2017); 10.1063/1.5017228 


\title{
Optimum efficiency control of a wind turbine with unknown desired trajectory and actuator faults
}

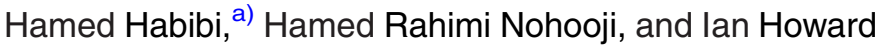 \\ Faculty of Science and Engineering, School of Civil and Mechanical Engineering, \\ Curtin University, Perth, Australia
}

(Received 5 September 2017; accepted 16 November 2017; published online 11 December 2017)

\begin{abstract}
The operational wind turbine efficiency in the power maximization regions and reliability improvement to reduce the produced power cost can both be enhanced by using an appropriate controller to cope with the highly nonlinear behavior of wind turbines in the presence of wind speed variation and actuator faults. In this regard, a nonlinear controller is proposed to make the wind turbine operate effectively despite some of the actuator faults, similar to the fault-free case. The considered actuator faults are pitch and generator actuator biases, as well as pitch actuator dynamic change, including pump wear, hydraulic leak, and high air content in the oil. Also, the wind speed is assumed to be an unmeasurable disturbance, and, accordingly, when using the neural network scheme, the unknown desired trajectory is reconstructed, so that the captured power is maximized. The proposed controller is shown to be able to keep the wind turbine tracking the reconstructed desired trajectory with sufficient accuracy. By using the Lyapunov analysis, the boundedness of the closed-loop system with the proposed controller is proven. The designed controller is verified via numerical simulations. The effectiveness of the proposed controller is evaluated in comparison with industrial constant gain controller results. Published by AIP Publishing. https://doi.org/10.1063/1.5003380
\end{abstract}

\section{INTRODUCTION}

Nowadays, the harvesting of clean electrical energy from wind by utilising wind turbines is becoming more industrialised to respond to the increasing energy demand as well as to reduce usage of constantly decreasing fossil fuels and their consequential environmental pollution. ${ }^{1}$ Modern wind turbines are mostly designed with long blades to increase the blade swept area and are often located in remote locations, e.g., the middle of oceans, to encounter higher accessible wind speed, which, consequently, leads to more captured energy. ${ }^{2,3}$ However, the performance of larger offshore wind turbines when operating in harsh environments is more likely to be downgraded, i.e., have reduced extracted energy due to abnormalities in the structure and mechanisms, which are addressed as faults. ${ }^{4}$ This issue leads to an additional need for maintenance procedures and longer shutdown maintenance periods, as well as an increased final price of the produced power. When considering the above-mentioned issues, several control schemes have been implemented on wind turbines in order to keep wind turbines operating at the desired trajectory and to be tolerant of the faults, which is called fault-tolerant control (FTC). ${ }^{1}$

In terms of the FTC design, the controller is designed to operate satisfactorily in fault-free situations as well as to remove the fault effects from the overall system performance to keep it as close as possible to the fault-free performance, until the next prescheduled maintenance activity can be performed. ${ }^{5}$ Also, it is advantageous to determine the fault information, including fault time, period, size, and location, either for supervisory control or to be used in the maintenance procedure. ${ }^{5,6}$ The robustness of the overall FTC scheme against system noise, model uncertainty, and unmeasured exogenous disturbances is challenging for complicated

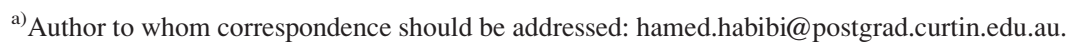


systems with different sources of noise and disturbance, such as wind turbines. Indeed, system noise and disturbance can mistakenly be seen as faults on the FTC scheme. ${ }^{7,8}$

The application of the FTC on wind turbines has emerged in the past decade, and several approaches have been addressed. ${ }^{1}$ Also, a benchmark model of the wind turbine was proposed in Ref. 9 and which was the basis of an international wind turbine FTC design announcement, and the results regarding faults in the pitch system, the gearbox, the generator, and the converter system, are summarized in Ref. 1. The highly nonlinear behaviour of wind turbines is one of the most challenging issues regarding their FTC designs, which mostly leads to approaches to linearize the model around several operating points. ${ }^{10}$ In Ref. 7, a FTC for pitch actuator faults was designed for a linearized wind turbine model utilising the robust linear parameter varying controller. When using several controllers, each of them being designed for a specific operational point and, when switching among them, may decrease the overall system performance. ${ }^{7}$ So, it is desirable to capture the whole nonlinear behaviour of wind turbines by utilizing a nonlinear controller design. In Ref. 11, the latent sensor and actuator faults that use the baseline controller were considered, and an extended sliding mode observer to estimate states as well as faults was designed by using linear matrix inequality. Also, in Ref. 12, the set membership approach was used, which contains consistent states with the measurement from a wind turbine, including model uncertainties and noises, with a subsequent deviation of the measured signals from the corresponding set being translated as a fault. By using this approach, a family of analytical redundancy relations for the whole wind turbine model, including uncertainties, was proposed. ${ }^{13}$ In similar studies, the blade root moment sensor additive and multiplicative faults, ${ }^{14}$ pitch sensor faults, and pitch actuator faults ${ }^{15}$ were also considered. Finally, the application of a fuzzy inference system and a fuzzy nonlinear system representation on the wind turbine has recently emerged to deal with model nonlinearity as well as to design the fuzzy gain scheduling the FTC scheme. ${ }^{16}$

The wind turbine operational region at the control system level can be divided into two different regions, which include the so-called partial load region and the full load region, ${ }^{17}$ such that, in the former, the aim is to maximize the captured energy, whereas, in the latter, despite the higher available energy content in the wind, the aim is to retain the produced power at its nominal value to protect the wind turbine structure from a catastrophic operation that will induce more stress on the structure and, consequently, may damage it. ${ }^{18,19}$ Tracking of the maximum power point is of interest in the partial load operation to keep the produced power as close as possible to the optimum extractable wind power. ${ }^{20}$ The wind speed is highly stochastic, and its accurate measurement, by using anemometers located at the top of the turbine hub, is not possible because of the temporal and spatial distribution of the wind speed over the blade plane. ${ }^{21}$ So, in a partial load operation, it is not possible to define the desired trajectory on the basis of wind speed. Accordingly, different methods have been deployed to estimate wind speed, e.g., unknown input observer, ${ }^{7}$ Kalman filter observer, ${ }^{22,23}$ and radial basis function neural networks (RBFNN). ${ }^{6,24}$

Motivated by the aforementioned issues, in this paper, the desired trajectory and aerodynamic torque, which are not known a priori, are reconstructed by using a RBFNN-based scheme as part of the proposed controller. Also, to let the proposed controller be applicable in practice, the whole nonlinear model of a wind turbine, including actuator dynamics, is utilized. The nonlinear adaptive controller is designed to be robust against the actuators dynamic change and bias, while making the wind turbine operate on an optimum operation trajectory with sufficient accuracy, despite the presence of noise and high wind speed variation. By using the Lyapunov analysis, the boundedness of all closed-loop system signals is ensured. Finally, the proposed controller performance is compared with an available industrial constant gain controller (ICGC) to evaluate its effectiveness. Therefore, compared with others' work, the contributions of this paper can be summarized as (a) construction of the desired trajectory in the proposed controller; (b) use of a practical model to capture the whole nonlinear behavior of the wind turbine, including both generator and pitch actuator faults in the model; and (c) the estimation of unknown actuator faults, which are used in the proposed controller structure. As well as assisting manual maintenance procedures, the effectiveness of the proposed controller has been evaluated by using both theoretical and numerical methods. 
The rest of this paper is as follows. The wind turbine model is presented in Sec. II. Control objectives, ICGC, and some technical lemmas are presented in Sec. III. The proposed generator torque and pitch controllers are given in Sec. IV. The proposed controllers are simulated numerically, and the results are illustrated in Sec. V, on which basis the discussions are stated. Finally, conclusions are given in Sec. VI.

\section{WIND TURBINE MODEL}

In this section, the benchmark model of the wind turbine is presented, which is an accepted model, including the possible faults, which are considered in this paper. ${ }^{25}$ The transferred power to rotor shaft, $P_{a}$, is stated as $P_{a}=C_{p}(\beta, \lambda) . P_{w}$, where $P_{w}$ and $C_{p}$ are the free wind power and the power coefficient, respectively. The power coefficient is a function of $\beta$ and $\lambda$, which are the blade pitch angle and tip speed ratio, respectively. Also, $P_{w}=0.5 \rho \pi R^{2} V_{r}^{3}$ and $\lambda=R \omega_{r} / V_{r}$, such that $R, \rho$, and $V_{r}$ are blade length, air density, and effective wind speed, respectively. The kinetic energy available in the wind is transferred into the rotor shaft and causes it to rotate with speed $\omega_{r}$. The effective wind speed causes an aerodynamic torque, $T_{a}$, and thrust, $F_{t}$, on the rotor as ${ }^{6}$

$$
\begin{aligned}
T_{a} & =\frac{1}{2} \rho \pi R^{3} V_{r}^{2} C_{q}(\beta, \lambda), \\
F_{t} & =\frac{1}{2} \rho \pi R^{2} V_{r}^{2} C_{t}(\beta, \lambda),
\end{aligned}
$$

where $C_{q}=C_{p} / \lambda$ and $C_{t}$ are torque and thrust coefficients, respectively, which are functions of $\beta$ and $\lambda$, as illustrated in Fig. 1 . Also $R$ is the blade length. The power coefficient, $C_{p}$, can be stated as a function of the tip speed ratio, $\lambda=R \omega_{r} / V_{r}$, and the pitch angle, $\beta$. In this paper, the empirical equation for $C_{p}$ is utilized as ${ }^{19,26}$

$$
C_{p}(\beta, \lambda)=C_{1}\left(\frac{C_{2}}{\lambda_{i}}-C_{3} \beta-C_{4}\right) \exp \left(\frac{-C_{5}}{\lambda_{i}}\right)+C_{6} \lambda, \quad \frac{1}{\lambda_{i}}=\frac{1}{\lambda+0.08 \beta}-\frac{0.035}{\beta^{3}+1},
$$

where $C_{1}=0.5176, C_{2}=116, C_{3}=0.4, C_{4}=5, C_{5}=21$, and $C_{6}=0.0068$.

The tower is modelled by considering the applied thrust force, which leads to a fore-aft motion of the nacelle, as

$$
M_{t} \ddot{x}_{t}=F_{t}-B_{t} \dot{x}_{t}-K_{t} x_{t}
$$

where $B_{t}, K_{t}$, and $M_{t}$ are the tower damping coefficient, bending stiffness coefficient, and top mass, respectively, and $x_{t}$ is the nacelle displacement from its equilibrium position. Accordingly, the effective wind speed at rotor plane, $V_{r}$, can be expressed as free wind speed, $V_{w}$, and nacelle displacement, as $V_{r}=V_{w}-\dot{x}_{t}{ }^{24}$

The blade shaft is directly connected to a drivetrain to increase the rotational speed to be fed into the power generator. The drivetrain is modelled as a two-mass system, which is shown
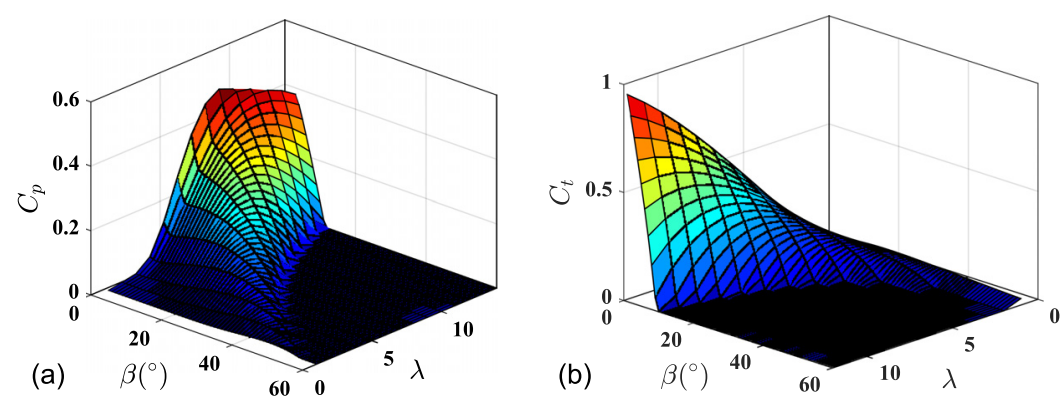

FIG. 1. (a) Power coefficient and (b) thrust coefficient. 
in Fig. 2, where $J_{r}$ and $J_{g}$ are rotor and generator inertia, respectively. The drivetrain includes $K_{d t}$ and $B_{d t}$, which are torsional stiffness and damping, respectively, which leads to the main shaft torsional angle of twist, i.e., $\theta_{\Delta}$. The drivetrain speed ratio and efficiency are $N_{g}$ and $\eta_{d t}$, respectively, and $T_{g}$ is the generator shaft torque, which is rotating at speed $\omega_{g}$. Finally, viscous frictions, $B_{g}$ and $B_{r}$, are considered on the rotor and generator shafts. ${ }^{6,7}$ In Fig. $2, T_{l}$ and $T_{h}$ are rotor and generator shafts exciting and load torques, respectively. The numerical value of the wind turbine parameters are available in the literature. ${ }^{7}$ The drivetrain dynamics equations can be derived as ${ }^{6}$

$$
\begin{aligned}
& J_{r} \dot{\omega}_{r}(t)=T_{a}(t)-K_{d t} \theta_{\Delta}(t)-\left(B_{r}+B_{d t}\right) \omega_{r}(t)+\frac{B_{d t}}{N_{g}} \omega_{g}(t), \\
& J_{g} \dot{\omega}_{g}(t)=\frac{\eta_{d t} K_{d t}}{N_{g}} \theta_{\Delta}(t)+\frac{\eta_{d t} B_{d t}}{N_{g}} \omega_{r}(t)-\left(B_{g}+\frac{\eta_{d t} B_{d t}}{N_{g}{ }^{2}}\right) \omega_{g}(t)-T_{g}(t), \quad \dot{\theta}_{\Delta}(t)=\omega_{r}(t)-\frac{1}{N_{g}} \omega_{g}(t) .
\end{aligned}
$$

The rotor and generator speed and its rate sensors are manipulated by noise as $\omega_{r, s}=\omega_{r}$ $+\nu_{\omega_{r}}, \omega_{g, s}=\omega_{g}+\nu_{\omega_{g}}$, and $\dot{\omega}_{g, s}=\dot{\omega}_{g}+\nu_{\dot{\omega}_{g}}$, respectively, where $\nu_{\omega_{r}}, \nu_{\omega_{g}}$, and $\nu_{\dot{\omega}_{g}}$ are additive noises.

The pitch angle of the blades is adjusted to the reference value, i.e., $\beta_{\text {ref }}$, by using the pitch actuator, which is a piston servo hydraulic mechanism that can be modelled as a second-order system as ${ }^{1}$

$$
\ddot{\beta}+2 \tilde{\omega}_{n} \tilde{\xi} \dot{\beta}+\tilde{\omega}_{n}^{2} \beta=\tilde{\omega}_{n}^{2} \beta_{r e f},
$$

where $\tilde{\xi}$ and $\tilde{\omega}_{n}$ are the pitch mechanism damping ratio and the natural frequency, respectively. When considering a practical controller of the wind turbine, the operational ranges of the pitch actuator are considered as $\dot{\beta}_{\min } \leq \dot{\beta} \leq \dot{\beta}_{\max }, \beta_{\min } \leq \beta \leq \beta_{\max }$. Note that $(\bullet)_{\max }$ and $(\bullet)_{\min }$ stand for maximum and minimum possible value, respectively, for variable $(\bullet)$. The pitch angle and its rate sensors are $\beta_{s}=\beta+\nu_{P s}$ and $\dot{\beta}_{s}=\dot{\beta}+\nu_{\dot{\beta}}$, respectively, where $\nu_{P s}$ and $\nu_{\dot{\beta}}$ are noise contents. It should be noted that the blades are considered as rigid bodies, such that their flexible bending oscillations, including flapwise and edgewise, are ignored. ${ }^{10}$

The pitch actuator dynamic change reduces the speed of the response, which has a drastic effect on power regulation of wind turbines. ${ }^{7}$ Pump wear, hydraulic leak, and high air content in oil are the most commonly reported dynamic changes of the pitch mechanism, which cause the slower response. The pitch actuator damping ratio and natural frequency for the dynamic change situation are shown in Table I. ${ }^{2,7}$ It should be noted that $N, P W, H L$, and $H A C$ stand for normal, pump wear, hydraulic leaks, and high air content situations, respectively. Also, $\omega_{n, X}$ and $\xi_{X}$ are the natural frequency and the damping ratio, respectively, in situation $X$, and $\alpha_{f_{1}}$ and $\alpha_{f_{2}}$ are fault indicators. In Fig. 3, the effect of pump wear, hydraulic leak, and high air content are illustrated, where it is obvious that the changed dynamic response is slower than the normal response. However, the pitch angle bias, i.e., $f_{P S}$, is introduced into the pitch model to consider the effect of this additive actuator fault, ${ }^{16,27}$ which may lead the pitch angle $\beta$ to deviate to $\beta+f_{P s}$. This fault causes an unbalanced rotor rotation, which increases the drivetrain fatigue probability. ${ }^{16}$ Pitch actuator bias is possible in a partial load operation of the wind turbine

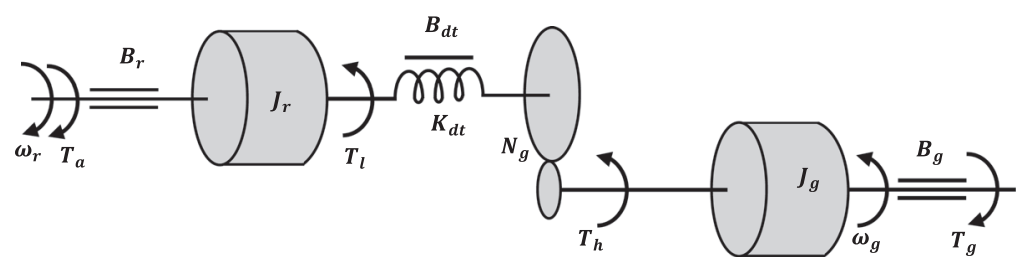

FIG. 2. Drivetrain mechanism. 
TABLE I. Pitch actuator parameters in dynamic change situation.

\begin{tabular}{lccc}
\hline \hline & Natural frequency $(\mathrm{rad} / \mathrm{s})$ & Damping ratio & Fault indicator \\
\hline Normal & $\omega_{n, N}=11.11$ & $\xi_{N}=0.6$ & $\alpha_{f_{1}}=\alpha_{f_{2}}=0$ \\
Pump wear & $\omega_{n, P W}=7.27$ & $\xi_{P W}=0.75$ & $\alpha_{f_{1}}=0.6316, \alpha_{f_{2}}=0.29688$ \\
Hydraulic leak & $\omega_{n, H L}=3.42$ & $\xi_{H L}=0.9$ & $\alpha_{f_{1}}=1, \alpha_{f_{2}}=0.87853$ \\
High air content & $\omega_{n, H A C}=5.73$ & $\xi_{H A C}=0.45$ & $\alpha_{f_{1}}=0.81083, \alpha_{f_{2}}=1$ \\
\hline \hline
\end{tabular}

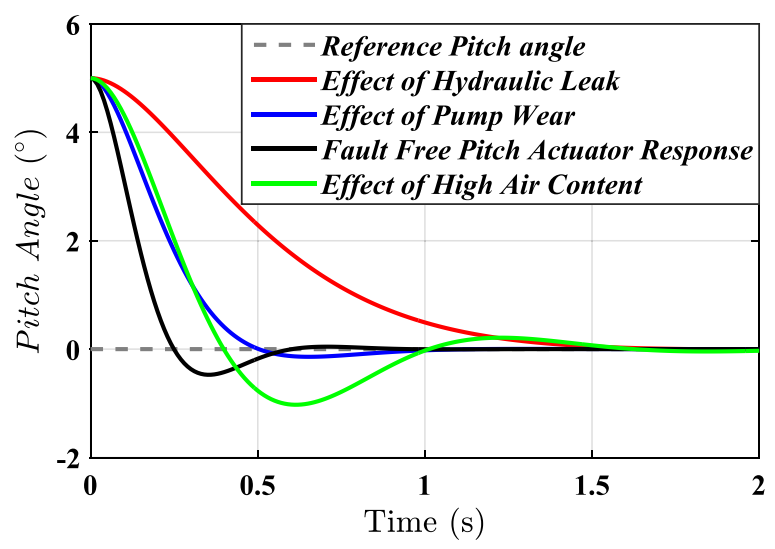

FIG. 3. Pitch mechanism response in different situations.

because of pitch actuator malfunction, which implies the need for the controller to remove its effect. The pitch mechanism dynamic change is modelled as a convex function of natural frequencies and damping ratios. ${ }^{2}$ When considering pitch actuator bias and dynamic change, according to Table I, the pitch actuator model can be rewritten as follows: ${ }^{18}$

$$
\begin{aligned}
\ddot{\beta} & =-\omega_{n, N}{ }^{2}\left(\beta+f_{P S}\right)-2 \omega_{n, N} \xi_{N} \dot{\beta}+\omega_{n, N}{ }^{2} \beta_{r e f}+\Delta \tilde{f}_{P A D}, \\
\Delta \tilde{f}_{P A D} & =-\alpha_{f_{1}} \Delta\left(\tilde{\omega}_{n}^{2}\right) \beta-2 \alpha_{f_{2}} \Delta\left(\tilde{\omega}_{n} \tilde{\xi}\right) \dot{\beta}+\alpha_{f_{1}} \Delta\left(\tilde{\omega}_{n}{ }^{2}\right) \beta_{r e f}, \\
\tilde{\omega}_{n}{ }^{2} & =\omega_{n, N}{ }^{2}+\alpha_{f_{1}} \Delta\left(\tilde{\omega}_{n}{ }^{2}\right), \quad \Delta\left(\tilde{\omega}_{n}{ }^{2}\right)=\omega_{n, H L}{ }^{2}-\omega_{n, N}{ }^{2}, \\
\tilde{\omega}_{n} \tilde{\xi} & =\omega_{n, N} \xi_{N}+\alpha_{f_{2}} \Delta\left(\tilde{\omega}_{n} \tilde{\xi}\right), \quad \Delta\left(\tilde{\omega}_{n} \tilde{\xi}\right)=\omega_{n, H A C} \xi_{H A C}-\omega_{n, N} \xi_{N} .
\end{aligned}
$$

The shaft kinetic energy is transferred to electrical energy in the generator, which is modelled as a delayed first-order system with time delay, $\tau_{g}$, and should track the reference load torque, $T_{g, r e f}$, demanded by the generator controller as

$$
\dot{T}_{g}=-a_{g} T_{g}+a_{g} T_{g, r e f}+\Delta f_{G C},
$$

where $a_{g}=1 / \tau_{g}$. It is assumed that the generator torque bias, i.e., $f_{T_{g}}$, is considered in the model, which may cause the generator torque to deviate to $T_{g}+f_{T_{g}}{ }^{13}$ The generator torque bias is combined in the generator model as $\Delta f_{G C}=-a_{g} f_{T_{g}}{ }^{12}$ Also, the measured generator torque is $T_{g, s}=T_{g}+\nu_{T_{g, s}}$, where $\nu_{T_{g, s}}$ is the noise content. ${ }^{12}$ In addition, the generator torque and its derivative are considered to be limited, as $\dot{T}_{g, \min } \leq \dot{T}_{g} \leq \dot{T}_{g, \max }, T_{g, \min } \leq T_{g} \leq T_{g, \max }$.

Finally, the power convertor, and its internal controller, is assumed to be faster than the nominal controller of the wind turbine and, consequently, is modelled as a static relation, $P_{a}=\eta_{g} T_{g} \omega_{g}$, where $\eta_{g}$ is the generator efficiency. ${ }^{13}$ It is also assumed that the blades are always perpendicular to the wind direction and, therefore, the yaw mechanism is not considered. 


\section{WIND TURBINE CONTROL PRELIMINARIES}

In this section, the wind turbine control objectives and performance criteria are introduced. Also, to evaluate the proposed controller numerically, ICGC, which is one of the most applied industrial controllers in a partial load operation, ${ }^{1,7,20}$ is explained. Finally, some lemmas are stated to be used in the proposed controller design.

\section{A. Objectives}

At the control system level, the wind turbine operation can be divided into two distinguishable regions, the so-called partial load region, i.e., extracting maximum power, and the full load region, i.e., regulation for nominal power. These two regions are shown in Fig. 4, which is the so-called ideal power curve, where $V_{r, \text { cut-in }}$ and $V_{r, \text { cut-out }}$ are maximum and minimum operational wind speeds, respectively, such that, for wind speeds out of this region, the wind turbine is shut down because, for wind speed less than $V_{r, c u t-i n}$, the produced power does not cover the operational cost, and, after $V_{r, \text { cut-out }}$, despite the higher available power in the wind, the wind turbine operation will lead to catastrophic structural damage. ${ }^{28}$ Also, the wind turbine produces its nominal power, i.e., $P_{a, N}$, at $V_{r, N}$. The control objective in the partial load operation, which is from $V_{r, \text { cut-in }}$ to $V_{r, N}$ and is of interest in this paper, requires tuning all control inputs to maximize the produced power, whereas, in a full load operation, these inputs should make the wind turbine produce its nominal power, $P_{a, N}$. When considering the generated power, i.e., $P_{a}=C_{p}(\beta, \lambda) \cdot P_{w}$ and the wind speed, $V_{r}$, which is considered as a disturbance, it can be concluded that, if the power coefficient, $C_{p}$, is maximized, it will lead to harvesting the most available power from the wind. According to (2), it can be seen that $C_{p, \max }=0.48$, which is at $\beta_{\text {opt }}=0^{\circ}$ and $\lambda_{\text {opt }}=8.1 .^{19}$ However, to keep the power coefficient at its maximum value, the tip speed ratio, $\lambda$, should be kept at $\lambda_{\text {opt }}=8.1$. When considering $\lambda=R \omega_{r} / V_{r}$, the desired rotor speed, i.e., $\omega_{r, \text { desired }}$, can be stated as a function of wind speed as $\omega_{r, \text { desired }}=\lambda_{\text {opt }} V_{r} / R{ }^{6}$ To fulfill the control objective, the load generator torque, $T_{g, r e f}$, is controlled such that, via the drivetrain dynamics, the rotor speed tracks $\omega_{r, \text { desired. }}{ }^{25}$

Remark 1. There may be an operational scheme in which the pitch actuator is not active in the partial load operation and pitch angle has to be fixed at the optimal value, i.e., $\beta_{\text {opt }}=0^{\circ}$. Consequently, there is no need for the pitch controller to be designed. The wind speed is a randomly varying disturbance, and it cannot be guaranteed that the wind turbine is only operating in the partial load region. Actually, the wind turbine operational region can rapidly and continuously vary from the partial to the full load region and vice versa. Therefore, it is important to guarantee, regardless of the operation region, that the pitch actuator follows the desired pitch angle accurately, whose dynamic behaviour might have changed. Also, due to this excessive operational range variation, a pitch bias may be added into the pitch actuator. So, it is proposed that a controller such that, despite the presence of a dynamic change or actuator bias, it keeps the pitch angle at the requested value, which is either zero in the partial load region or should be tuned in the full load region, in which a simple industrial Proportional Integral (PI) controller can be implemented. ${ }^{2}$ In fact, the proposed pitch controller is readily designed to be used in the full load region as an extension of the current study, and, therefore, just the desired pitch angle should be constructed separately.

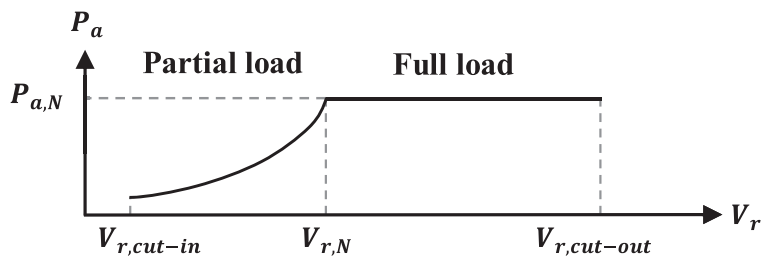

FIG. 4. Ideal power curve. 


\section{B. Criteria}

The evaluation of wind turbine performance is studied by using numerical criteria to quantify the proposed controller performance. The total captured energy, i.e., $E$, is expected to be as close as possible to the optimal one, i.e., $E_{\text {optimal }}$. Therefore, the efficiency is calculated as the first criterion as, Efficiency $=E / E_{\text {optimal }}$, where $E=\int P_{a} d t$ and $E_{\text {optimal }}=\int P_{\text {maximum }} d t$, where $P_{\text {maximum }}=0.5 C_{p, \max } \rho \pi R^{2} V_{r}^{3}$. Also, the change rate of induced torsion angle on the main shaft of the drivetrain can be used as an index to represent the drivetrain shaft $\operatorname{stress}^{20}$ as a second criterion as, $S=\int \dot{\theta}_{\Delta}^{2} d t$. Also, $G T V=\int\left|\dot{T}_{g}\right| d t$ is used to compare the generator torque variation, commanded by the controller, which is highly related to wind speed variation and may lead to actuator rate saturation and failure. Finally, maximum and standard deviation values of generator torque, i.e., $\operatorname{Max}\left(T_{g}\right)$ and $\operatorname{STD}\left(T_{g}\right)$, respectively, are also considered to study generator controller behavior in actuator fault situations. ${ }^{22}$

\section{Reference controller}

In this paper, the ICGC is selected as the reference controller, which can be obtained by excluding the wind speed from the wind turbine dynamics. In the ICGC, the reference generator torque is tuned as ${ }^{7,20}$

$$
T_{g, r e f}=K \omega_{g}^{2},
$$

with $K=0.5 \rho A \eta_{d t} R^{3} C_{p, \max } / N_{g}^{3} \lambda_{\text {opt }}^{3}$, where, $C_{p, \max }=0.48$ and $\lambda_{\text {opt }}=8.1$, and the reference pitch angle is fixed at $\beta_{\text {ref }}=0^{\circ}$ to keep the power coefficient at the maximum power value.

\section{Technical lemmas}

Lemma 1. The state vector of a dynamic system $x$ is uniformly ultimately bounded (UUB) for the bounded initial condition, if there exists a positive definite Lyapunov function, $V(\boldsymbol{x}, t)$ satisfies $d V / d t<-b_{1} V+b_{2}$, where $b_{1}$ and $b_{2}$ are positive constants. Over time, $V(\boldsymbol{x}, t)$ stays in set $\Omega=\left\{V \mid V \leq b_{2} / b_{1}\right\}$.

Proof. Multiplying both sides of $d V / d t<-b_{1} V+b_{2}$ by $\exp \left(b_{1} t\right)$ leads to $\exp \left(b_{1} t\right) d V / d t$ $<-\exp \left(b_{1} t\right) b_{1} V+b_{2} \exp \left(b_{1} t\right)$, which is equivalent to $d\left[\exp \left(b_{1} t\right) V\right] / d t<\exp \left(b_{1} t\right) b_{2}$. Now, when taking the integral with respect to time from both sides leads to $\exp \left(b_{1} t\right) V-V(0)$ $<\exp \left(b_{1} t\right) b_{2} / b_{1}-b_{2} / b_{1}$. Multiplying by $\exp \left(-b_{1} t\right)$, gives $V<b_{2} / b_{1}+\exp \left(-b_{1} t\right)$ $\left[V(0)-b_{2} / b_{1}\right]$. However, for a bounded initial condition and consequent bounded $V(0), V$ is bounded. Consequently, $\boldsymbol{x}$ is bounded because $V$ includes a positive term as a function of states. However, over time, $\exp \left(-b_{1} t\right)$ approaches to zero. So, $V$ satisfies $V<b_{2} / b_{1} \cdot{ }^{29,30}$

Lemma 2. Define variable $\tilde{a}$, as $\tilde{a}=\hat{a}-a$, then $-2 \sigma_{d} \tilde{a} \hat{a} \leq-\sigma_{d} \tilde{a}^{2}+\sigma_{d} a^{2}$ holds true for any positive constant $\sigma_{d}$.

Proof. Considering $(\tilde{a}+a)^{2} \geq 0$ leads to $\tilde{a}^{2}+a^{2} \geq-2 \tilde{a} a$. Replace $-2 \tilde{a} a$ with $-2 \tilde{a}(\hat{a}-\tilde{a})$ and multiply both sides by $\sigma_{d}$. Consequently, $-2 \sigma_{d} \tilde{a} \hat{a} \leq-\sigma_{d} \tilde{a}^{2}+\sigma_{d} a^{2}$ can be obtained.

Lemma 3. $0 \leq|\xi|-\xi^{2} / \sqrt{\xi^{2}+\gamma^{2}}<\gamma$ holds true, for any variable $\xi$ and positive constant $\gamma$.

Proof. Consider the inequality $\xi^{4} \leq \xi^{2} \gamma^{2}+\xi^{4}$ and take the square root from both sides. Consequently, it proves the left side of the inequality. To prove the right-hand side, consider $0 \leq 2|\xi| \gamma$ and add $\xi^{2}+\gamma^{2}$ to both sides. Taking the square root of the resulted inequality leads to $\sqrt{\xi^{2}+\gamma^{2}} \leq \gamma+|\xi|$. Accordingly, it can be seen that $-1 / \sqrt{\xi^{2}+\gamma^{2}} \leq-1 /(\gamma+|\xi|)$ which leads to $|\xi|-\xi^{2} / \sqrt{\xi^{2}+\gamma^{2}} \leq|\xi|-\xi^{2} /(\gamma+|\xi|)$. Finally, when considering $|\xi|-\xi^{2} /(\gamma+|\xi|)$ $=\gamma|\xi| /(\gamma+|\xi|)$ and $\gamma|\xi| /(\gamma+|\xi|)<\gamma$, it can be seen that $|\xi|-\xi^{2} / \sqrt{\xi^{2}+\gamma^{2}}<\gamma$.

\section{WIND TURBINE OPTIMUM POWER POINT TRACKING FTC DESIGN}

In this section, the proposed controller is designed to track the maximum power coefficient, which leads to increasing the captured energy. First, the unknown desired trajectory is 
reconstructed by using neural networks, on which basis the generator torque controller is then designed. Finally, the pitch actuator controller is designed.

\section{A. Unknown desired trajectory construction}

As stated earlier, the effective wind speed at the rotor plane, $V_{r}$, is not accurately measurable when using the anemometer placed at the top of the nacelle, ${ }^{12}$ and, meanwhile, the desired rotor speed, to capture the most possible energy, is stated in terms of wind speed as

$$
\begin{aligned}
\omega_{r, \text { desired }} & =\frac{\lambda_{\text {opt }} V_{r}}{R}, \\
\omega_{g, \text { desired }} & =N_{g} \omega_{r, \text { desired }} .
\end{aligned}
$$

So, the desired trajectory is not known a priori. Accordingly, in this section, by exploiting the universal approximation capabilities of neural networks, the desired trajectory is reconstructed. ${ }^{30,31}$ When considering the well-developed approximation theory, ${ }^{30}$ the approximated desired trajectory, $\omega_{g, d}^{*}$, is expressed as a continuous function as,

$$
\omega_{g, d}^{*}=\boldsymbol{\Psi}^{* T}(t) \boldsymbol{\varphi}(t)+\varrho(t),
$$

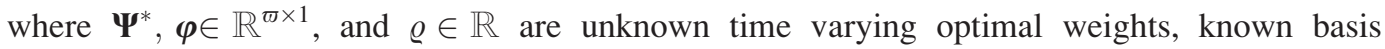
bounded functions, and unknown approximation errors, respectively. Also, $\varpi$ is the number of neurons in the neural network. Because $\boldsymbol{\Psi}^{*}$ and $\varrho$ are unknown, it is obvious that $\omega_{g, d}^{*}$ is still not usable in the controller design procedure. Therefore, the approximated desired trajectory, $\hat{\boldsymbol{\omega}}_{\mathrm{g}, d}$, is computed as

$$
\hat{\omega}_{g, d}=\hat{\boldsymbol{\Psi}}^{T}(t) \boldsymbol{\varphi}(t),
$$

where, $\hat{\boldsymbol{\Psi}}$ is the estimation of $\boldsymbol{\Psi}^{*}$, with the estimation error, $\tilde{\boldsymbol{\Psi}}=\boldsymbol{\Psi}^{*}-\hat{\boldsymbol{\Psi}}$.

Assumption 1. It is assumed that $|\varrho| \leq \varrho_{1},|\varrho| \leq \varrho_{2},\left\|\boldsymbol{\Psi}^{*}\right\| \leq \psi_{1}$ and $\left\|\dot{\boldsymbol{\Psi}}^{*}\right\| \leq \psi_{2}$, where $0<\varrho_{1}, \varrho_{2}, \psi_{1}, \psi_{2}<\infty$ are unknown constants, ${ }^{30}$ and $\|\cdot\|$ denotes the Euclidean norm operator for vectors.

The tracking error and its time derivative, which will be used in Sec. IV B to design the generator controller, are defined as $e_{\omega_{g}}=\hat{\omega}_{g, d}-\omega_{g, s}$ and $\dot{e}_{\omega_{g}}=\dot{\hat{\omega}}_{g, d}-\dot{\omega}_{g, s}$, where $\omega_{g, s}$ and $\dot{\omega}_{g, s}$ are the measured generator speed and its derivative sensors, respectively. Also, $\dot{\hat{\omega}}_{g, d}$ is obtained by considering (11) as

$$
\dot{\hat{\omega}}_{g, d}=\dot{\hat{\boldsymbol{\Psi}}}^{T}(t) \boldsymbol{\varphi}(t)+\hat{\boldsymbol{\Psi}}^{T}(t) \dot{\boldsymbol{\varphi}}(t) .
$$

Now, the adaption law for $\hat{\boldsymbol{\Psi}}$ is proposed as

$$
\dot{\hat{\boldsymbol{\Psi}}}=-\sigma \hat{\boldsymbol{\Psi}}(t)+\Lambda \boldsymbol{\eta}\left(e_{\omega_{g}}\right),
$$

where $\sigma$ and $\Lambda$ are positive design parameters, satisfying $\sigma>\Lambda+1$, and $\boldsymbol{\eta}\left(e_{\omega_{g}}\right) \in \mathbb{R}^{\varpi \times 1}$ is an arbitrarily selected bounded function, as $\boldsymbol{\eta}\left(e_{\omega_{g}}\right) \leq \eta_{1}$, where $\eta_{1}$ is an unknown positive constant. When considering (13), it can easily be shown that

$$
\ddot{\hat{\boldsymbol{\Psi}}}=\sigma^{2} \hat{\boldsymbol{\Psi}}(t)-\sigma \Lambda \boldsymbol{\eta}\left(e_{\omega_{g}}\right)+\Lambda \dot{\boldsymbol{\eta}}\left(e_{\omega_{g}}\right),
$$

where, $\dot{\boldsymbol{\eta}}\left(e_{\omega_{g}}\right)=\left(\partial \boldsymbol{\eta} / \partial e_{\omega_{g}}\right) \dot{e}_{\omega_{g}}$ and $\left\|\partial \boldsymbol{\eta} / \partial e_{\omega_{g}}\right\|$ is bounded. ${ }^{30}$

Theorem 1. When considering the adaption law (13), $\hat{\boldsymbol{\Psi}}$ and $\dot{\hat{\boldsymbol{\Psi}}}$ are UUB.

Proof. Choose a positive definite Lyapunov function, as

$$
V^{\omega_{g, d}}=\frac{1}{2} \tilde{\boldsymbol{\Psi}}^{T} \tilde{\boldsymbol{\Psi}}
$$


The time derivative of $V^{\omega_{g, d}}$ can be obtained as

$$
\dot{V}^{\omega_{g, d}}=\tilde{\boldsymbol{\Psi}}^{T} \dot{\boldsymbol{\Psi}}^{*}+\sigma \tilde{\boldsymbol{\Psi}}^{T} \boldsymbol{\Psi}^{*}-\sigma \tilde{\boldsymbol{\Psi}}^{T} \tilde{\boldsymbol{\Psi}}-\Lambda \tilde{\boldsymbol{\Psi}}^{T} \boldsymbol{\eta}
$$

When considering the inequality for the two vectors $\boldsymbol{A}, \boldsymbol{B} \in \mathbb{R}^{n \times 1}$ as, $\pm \boldsymbol{A}^{\boldsymbol{T}} \boldsymbol{B} \leq\left(\boldsymbol{A}^{\boldsymbol{T}} \boldsymbol{A}+\boldsymbol{B}^{T} \boldsymbol{B}\right) / 2$ and, $\boldsymbol{A}^{T} \boldsymbol{A}=\|\boldsymbol{A}\|^{2}$, the following can be derived from (16), as

$$
\begin{aligned}
\dot{V}^{\omega_{g, d}} & \leq\left(\tilde{\boldsymbol{\Psi}}^{T} \tilde{\boldsymbol{\Psi}}+\dot{\boldsymbol{\Psi}}^{* T} \dot{\boldsymbol{\Psi}}^{*}\right) / 2+\sigma\left(\tilde{\boldsymbol{\Psi}}^{T} \tilde{\boldsymbol{\Psi}}+\boldsymbol{\Psi}^{* T} \boldsymbol{\Psi}^{*}\right) / 2-\sigma \tilde{\boldsymbol{\Psi}}^{T} \tilde{\boldsymbol{\Psi}}+\Lambda\left(\tilde{\boldsymbol{\Psi}}^{T} \tilde{\boldsymbol{\Psi}}+\boldsymbol{\eta}^{T} \boldsymbol{\eta}\right) / 2 \\
& \leq \tilde{\boldsymbol{\Psi}}^{T} \tilde{\boldsymbol{\Psi}} / 2-\sigma \tilde{\boldsymbol{\Psi}}^{T} \tilde{\boldsymbol{\Psi}} / 2+\Lambda \tilde{\boldsymbol{\Psi}}^{T} \tilde{\boldsymbol{\Psi}} / 2+\left\|\dot{\boldsymbol{\Psi}}^{*}\right\|^{2}+\sigma\left\|\boldsymbol{\Psi}^{*}\right\|^{2}+\Lambda\|\boldsymbol{\eta}\|^{2}<-\Upsilon_{1} V^{\omega_{g, d}}+\Upsilon_{2},
\end{aligned}
$$

where, $\Upsilon_{1}=-1+\sigma-\Lambda$ and $\Upsilon_{2}=\sigma \psi_{1}^{2}+\psi_{2}^{2}+\Lambda \eta_{1}^{2}$. Since $0<\sigma, 0<\Lambda$, and according to $\sigma>\Lambda+1$, then $0<\Upsilon_{1}$ and $0<\Upsilon_{2}$. Now, when considering Lemma $1, \tilde{\boldsymbol{\Psi}}$ and $\hat{\boldsymbol{\Psi}}$ are UUB, and. also, when considering (13) and Assumption 1, it can be seen that $\hat{\boldsymbol{\Psi}}$ and consequently $\tilde{\boldsymbol{\Psi}}$ are UUB.

The desired trajectory construction diagram is illustrated in Fig. 5.

Now, after estimation of the unknown desired trajectory, $\hat{\omega}_{g, d}$, the exact tracking error and its time derivative, i.e., $e_{\omega_{g}}^{*}$ and $\dot{e}_{\omega_{g}}^{*}$, respectively, are defined as

$$
\begin{aligned}
& e_{\omega_{g}}^{*}=\omega_{g, d}^{*}-\omega_{g, s}, \\
& \dot{e}_{\omega_{g}}^{*}=\dot{\omega}_{g, d}^{*}-\dot{\omega}_{g, s} .
\end{aligned}
$$

When taking $e_{\omega_{g}}=\hat{\omega}_{g, d}-\omega_{g, s}$ and $\dot{e}_{\omega_{g}}=\dot{\hat{\omega}}_{g, d}-\dot{\omega}_{g, s}$ into consideration, $e_{\omega_{g}}^{*}$ and $\dot{e}_{\omega_{g}}^{*}$ can be rewritten as

$$
\begin{aligned}
& e_{\omega_{g}}^{*}=\omega_{g, d}^{*}-\hat{\omega}_{g, d}+e_{\omega_{g}}=\tilde{\boldsymbol{\Psi}}^{\boldsymbol{T}}(t) \boldsymbol{\varphi}(t)+\varrho(t)+e_{\omega_{g}}, \\
& \dot{e}_{\omega_{g}}^{*}=\dot{\tilde{\boldsymbol{\Psi}}}^{\boldsymbol{T}}(t) \boldsymbol{\varphi}(t)+\tilde{\boldsymbol{\Psi}}^{\boldsymbol{T}}(t) \dot{\boldsymbol{\varphi}}(t)+\underline{\varrho}(t)+\dot{e}_{\omega_{g}} .
\end{aligned}
$$

Therefore, when considering Theorem 1 and Assumption 1, it can be concluded that boundedness of $e_{\omega_{g}}$ and $\dot{e}_{\omega_{g}}$ leads to boundedness of $e_{\omega_{g}}^{*}$ and $\dot{e}_{\omega_{g}}^{*}$, respectively. So, in the sequel, it is aimed to design $T_{g, r e f}$, such that $\omega_{g, s}$ tracks as close as possible to the estimated desired generator speed, $\hat{\omega}_{g, d}$, such that $e_{\omega_{g}}$ and $\dot{e}_{\omega_{g}}$ are bounded.

\section{B. Generator torque controller design}

The design of the generator torque controller, $T_{g, r e f}$, to track the maximum power point, i.e., $\lambda_{o p t}=8.1$, is based on reducing the tracking error $e_{\omega_{g}}=\hat{\omega}_{g, d}-\omega_{g, s}$. In order to reduce the torsional angle of twist that may lead to drivetrain failure, it is desirable to keep the generator and rotor speed proportional through the gearbox ratio, ${ }^{22}$ accordingly, it is advantageous that $\dot{\theta}_{\Delta}=0$. So, in this paper, the controller is designed on the desirable operational mode of the wind turbine in which $\omega_{g}=N_{g} \omega_{r}{ }^{22}$ On this basis, the drivetrain model, (4), operating at the desired trajectory, can be rewritten as

$$
\dot{\omega}_{r}=a_{1} \omega_{r}+a_{2} \omega_{g}+a_{3} T_{a}, \quad \dot{\omega}_{g}=b_{1} \omega_{r}+b_{2} \omega_{g}+b_{3} T_{g},
$$

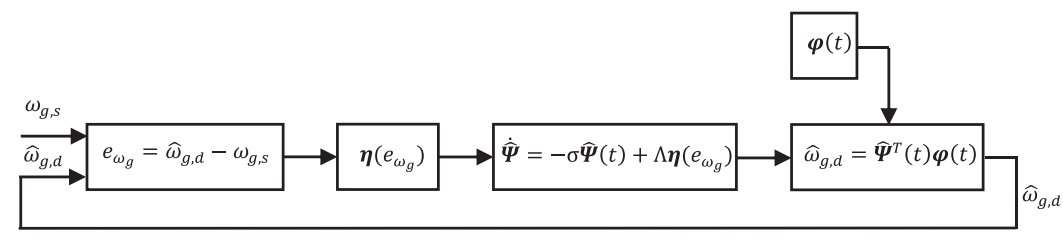

FIG. 5. Desired trajectory construction. 
where $\quad a_{1}=-\left(B_{d t}+B_{r}\right) / J_{r}, a_{2}=B_{d t} /\left(N_{g} J_{r}\right), a_{3}=1 / J_{r}, b_{1}=\eta_{d t} B_{d t} /\left(N_{g} J_{g}\right), b_{2}=\left(-\eta_{d t} B_{d t} /\right.$ $\left.N_{g}{ }^{2}-B_{g}\right) / J_{g}, b_{3}=-1 / J_{g}$. Accordingly, the second time derivative of the generator speed can be obtained as

$$
\ddot{\omega}_{g}=c_{1} \omega_{r}+c_{2} \omega_{g}+c_{3} T_{g}+c_{4} T_{a}+c_{5} T_{g, r e f}+d_{1}+f_{1},
$$

where $c_{1}=b_{1} a_{1}+b_{2} b_{1}, c_{2}=b_{1} a_{2}+b_{2}{ }^{2}, c_{3}=b_{2} b_{3}-a_{g} b_{3}, c_{4}=b_{1} a_{3}, c_{5}=b_{3} a_{g}, d_{1}=c_{1} \nu_{\omega_{r}}$ $+c_{2} \nu_{\omega_{g}}+c_{3} \nu_{T_{g, s}}$ and $f_{1}=b_{3} \Delta f_{G C}$.

Assumption 2. It is assumed that the accumulative fault, $f_{1}$, is bounded such that $\left|f_{1}\right| \leq \bar{f}_{1}$, where $\bar{f}_{1} \in \mathbb{R}^{+}$is an unknown constant. ${ }^{2}$ Indeed, when taking into account the maximum possible generator torque, it is assumed that the generator bias, i.e., $f_{T_{g}}$, is bounded, before the total failure of the generator, in which case, the maintenance procedure is inevitable. ${ }^{7}$ However, the time derivative of the fault is assumed to be bounded, i.e., $\left|\dot{f}_{1}\right| \leq \rho_{\dot{f}_{1}}$ where $\rho_{\dot{f}_{1}} \in \mathbb{R}^{+}$is an unknown constant. ${ }^{2}$ With regard to the accumulative disturbance, i.e., $d_{1}$, it is assumed that $\left|d_{1}\right| \leq \bar{d}_{1}$, where $\bar{d}_{1} \in \mathbb{R}^{+}$is an unknown constant, which is a reasonable practical issue. ${ }^{12}$

It is obvious that the aerodynamic torque, i.e., $T_{a}$, is contributing in (21) and, as stated earlier, the wind speed is not accurately measurable to compute the aerodynamic torque. Although by using (9) and (11), an estimation of wind speed and, consequently, aerodynamic torque, can be reconstructed, but, in this paper, to avoid computational complexity, a separate aerodynamic torque estimator is implemented. In this regard, a RBFNN is designed to estimate the aerodynamic torque, whose input vector is $Z=\left[T_{g}, \omega_{g}, \beta\right]^{T} \in \Omega_{Z}$ and, accordingly, the aerodynamic torque is given as

$$
T_{a}(\boldsymbol{Z})=\boldsymbol{\theta}^{*^{T}} \boldsymbol{h}(\boldsymbol{Z})+\varepsilon, T_{a, N N}(\boldsymbol{Z})=\boldsymbol{\theta}^{T} \boldsymbol{h}(\boldsymbol{Z})
$$

where $T_{a, N N}$ is a RBFNN approximation of $T_{a}, \boldsymbol{\theta}^{*} \in \mathbb{R}^{s}$ is an unknown optimal weight, $\boldsymbol{h}(\boldsymbol{Z})$ $=\left[h_{1}(\boldsymbol{Z}), h_{2}(\boldsymbol{Z}), \ldots, h_{s}(\boldsymbol{Z})\right]^{T} \in \mathbb{R}^{s}$, with $s>1$ being the neural network node number, and $h_{i}(\boldsymbol{Z})$ is selected as a Gaussian function, given by

$$
h_{i}(\boldsymbol{Z})=\exp \left(\frac{-\left(\boldsymbol{Z}-\boldsymbol{\vartheta}_{\boldsymbol{i}}\right)^{T}\left(\boldsymbol{Z}-\boldsymbol{\vartheta}_{\boldsymbol{i}}\right)}{\varphi_{i}^{2}}\right),
$$

where $\boldsymbol{\vartheta}_{i}=\left[\vartheta_{i, T_{g}}, \vartheta_{i, \omega_{g}}, \vartheta_{i, \beta}\right]^{T}$ is the center of the $i$ th input, and $\varphi_{i}$ is the width of the Gaussian function. $\boldsymbol{\theta}^{*}$ is defined as

$$
\boldsymbol{\theta}^{*}=\arg \min _{\boldsymbol{\theta} \in \mathbb{R}^{s}}\left[\sup _{\boldsymbol{Z} \in \Omega_{Z}}\left|T_{a}(\boldsymbol{Z})-T_{a, N N}(\boldsymbol{Z})\right|\right]
$$

and also $\varepsilon \in \mathbb{R}$ is an estimation error, which is bounded with an unknown bound $\bar{\varepsilon}>0$, such that, $|\varepsilon| \leq \bar{\varepsilon}$. $^{6,24}$

Now, to design the generator torque controller, a positive definite Lyapunov function is chosen as

$$
V_{1}^{T_{g}}=\frac{1}{2} e_{\omega_{g}}^{2},
$$

where its time derivative can be written as $\dot{\mathrm{V}}_{1}^{T_{g}}=e_{\omega_{g}} \dot{e}_{\omega_{g}}$. A variable transformation is defined as $e_{2, \omega_{g}}=\alpha_{\omega_{g}}-\dot{\omega}_{g, s}$, where $\alpha_{\omega_{g}}$ is the virtual control signal, designed as

$$
\alpha_{\omega_{g}}=\dot{\hat{\omega}}_{g, d}+k_{1, \omega_{g}} e_{\omega_{g}}
$$

where $k_{1, \omega_{g}}$ is a positive design parameter. Also, $\dot{\hat{\omega}}_{g, d}$ is obtained by using (12).

To reduce the tracking error of generator speed, the generator torque controller is proposed as 


$$
T_{g, \text { ref }}=\frac{1}{c_{5}}\left(g_{1}-c_{4} \hat{T}_{a}-\hat{f}_{1}+\frac{e_{2, \omega_{g}}}{\sqrt{e_{2, \omega_{g}}^{2}+\eta_{d_{1}}^{2}}} \hat{d}_{1}+k_{2, \omega_{g}} e_{2, \omega_{g}}\right),
$$

where $\eta_{d_{1}}$ and $k_{2, \omega_{2}}$ are positive design parameters, and $\hat{f}_{1}$ and $\hat{d}_{1}$ are estimations of $f_{1}$ and $d_{1}$, respectively. Also, $g_{1}=e_{\omega_{g}}+\dot{\alpha}_{\omega_{g}}-c_{1} \omega_{r, s}-c_{2} \omega_{g, s}-c_{3} T_{g, s}, \hat{T}_{a}=\hat{\boldsymbol{\theta}}^{T} \boldsymbol{h}$, and $\hat{\boldsymbol{\theta}}$ is the estimated weight of $\boldsymbol{\theta}^{*}$. Also, the adaptive laws are defined as

$$
\dot{\hat{d}}_{1}=\frac{e_{2, \omega_{g}}^{2}}{\sqrt{e_{2, \omega_{g}}^{2}+\eta_{d_{1}}^{2}}}-\sigma_{d_{1}} \hat{d}_{1}, \quad \dot{\hat{\boldsymbol{\theta}}}=\Gamma\left(-c_{4} e_{2, \omega_{g}} \boldsymbol{h}-\sigma_{c} \hat{\boldsymbol{\theta}}\right), \quad \dot{\hat{f}}_{1}=-e_{2, \omega_{g}}-\sigma_{f_{1}} \hat{f}_{1}, \quad \dot{\hat{\rho}}_{\tilde{f}_{1}}=-\sigma_{\tilde{f}_{1}} \hat{\rho}_{\tilde{f}_{1}}
$$

where $\sigma_{d_{1}}, \sigma_{c}, \sigma_{f_{1}}$, and $\sigma_{\tilde{f}_{1}}$ are positive design parameters. $\Gamma \in \mathbb{R}^{s}$ is the design matrix, such that $\Gamma=\Gamma^{T}>0$. Neural Network (NN) weight and disturbance estimation errors are $\tilde{\boldsymbol{\theta}}=$ $\hat{\boldsymbol{\theta}}-\boldsymbol{\theta}^{*}$ and $\tilde{d}_{1}=\hat{d}_{1}-D$, respectively, where $D$ is a positive unknown constant, satisfying $0<c_{4} \bar{\varepsilon}+\bar{d}_{1} \leq D$. Fault estimation error $\tilde{f}_{1}=\hat{f}_{1}-f_{1}$, which is assumed to be bounded as ${ }^{2}$ $\left|\tilde{f}_{1}\right|<\rho_{\tilde{f}_{1}}$, where $\rho_{\tilde{f}_{1}}$ is a positive unknown constant. So, the estimation of $\rho_{\tilde{f}_{1}}$ is defined as $\hat{\rho}_{\tilde{f}_{1}}$, and its estimation error is $\tilde{\rho}_{\tilde{f}_{1}}=\hat{\rho}_{\tilde{f}_{1}}-\rho_{\tilde{f}_{1}}$.

Theorem 2. Consider the wind turbine model (1)-(7) and the proposed generator torque controller (27). Let the initial conditions of drivetrain and generator dynamics be bounded. Then, for all pitch angles, generator speed tracking error, i.e., $e_{\omega_{g}}$, and its time derivative, i.e., $\dot{e}_{\omega_{g}}$, are UUB.

Proof. Choose a positive definite Lyapunov function as

$$
V^{T_{g}}=V_{1}^{T_{g}}+\frac{1}{2} e_{2, \omega_{g}}^{2}+\frac{1}{2} \tilde{d}_{1}^{2}+\frac{1}{2} \tilde{\boldsymbol{\theta}}^{T} \Gamma^{-1} \tilde{\boldsymbol{\theta}}+\frac{1}{2} \tilde{f}_{1}^{2}+\frac{1}{2} \tilde{\rho}_{\tilde{f}_{1}}^{2}+V^{\omega_{g, d}} .
$$

Taking the time derivative of (29) obtains $\dot{V}^{T_{g}}=\dot{V}_{1}^{T_{g}}+e_{2, \omega_{g}} \dot{e}_{2, \omega_{g}}+\tilde{d}_{1} \dot{\tilde{d}}_{1}+\tilde{\boldsymbol{\theta}}^{T} \Gamma^{-1} \dot{\tilde{\boldsymbol{\theta}}}+\tilde{f}_{1} \dot{\tilde{f}}_{1}$ $+\tilde{\rho}_{\tilde{f}_{1}} \dot{\tilde{\rho}}_{\tilde{f}_{1}}+\dot{V}^{\omega_{g, d}}$, which can be rewritten as

$$
\dot{V}^{T_{g}}=\sum_{i=1}^{4} \Pi_{i}^{T_{g}}-k_{1, \omega_{g}} e_{\omega_{g}}^{2}-k_{2, \omega_{g}} e_{2, \omega_{g}}^{2}+\dot{V}^{\omega_{g, d}}
$$

where $\Pi_{1}^{T_{g}}=-e_{2, \omega_{g}} c_{4} \varepsilon-e_{2, \omega_{g}} d_{1}+\left(\tilde{d}_{1}-\hat{d}_{1}\right) e_{2, \omega_{g}}^{2} / \sqrt{e_{2, \omega_{g}}^{2}+\eta_{d_{1}}^{2}}-\sigma_{d_{1}} \hat{d}_{1} \tilde{d}_{1}, \Pi_{2}^{T_{g}}=-c_{4} e_{2, \omega_{g}}\left(\tilde{\boldsymbol{\theta}}^{T}\right.$ $\left.+\boldsymbol{\theta}^{*^{T}}-\hat{\boldsymbol{\theta}}^{T}\right) \boldsymbol{h}-\sigma_{c} \tilde{\boldsymbol{\theta}}^{T} \hat{\boldsymbol{\theta}}, \quad \Pi_{3}^{T_{g}}=-e_{2, \omega_{g}}\left(-\hat{f}_{1}+f_{1}+\tilde{f}_{1}\right)-\sigma_{f_{1}} \hat{f}_{1} \tilde{f}_{1}-\dot{f}_{1} \tilde{f}_{1} \quad$ and $\quad \Pi_{4}^{T_{g}}=-\sigma_{\tilde{f}_{1}} \hat{\rho}_{\tilde{f}_{1}} \tilde{\rho}_{\tilde{f}_{1}}$. According to Lemmas 2 and 3 , it can be shown that

$$
\begin{aligned}
\Pi_{1}^{T_{g}} & <\left|e_{2, \omega_{g}}\right|\left(c_{4} \bar{\varepsilon}+\bar{d}_{1}\right)-\frac{e_{2, \omega_{g}}^{2}}{\sqrt{e_{2, \omega_{g}}^{2}+\eta_{d_{1}}^{2}}} D \\
& =\left(\left|e_{2, \omega_{g}}\right|-\frac{e_{2, \omega_{g}}^{2}}{\sqrt{e_{2, \omega_{g}}^{2}+\eta_{d_{1}}^{2}}}\right) D-\sigma_{d_{1}} \hat{d}_{1} \tilde{d}_{1}<\eta_{d_{1}} D-\frac{\sigma_{d_{1}}}{2} \tilde{d}_{1}^{2}+\frac{\sigma_{d_{1}}}{2} D^{2}, \\
\Pi_{2}^{T_{g}} & <-\frac{\sigma_{c}}{2} \tilde{\boldsymbol{\theta}}^{T} \tilde{\boldsymbol{\theta}}+\frac{\sigma_{c}}{2} \boldsymbol{\theta}^{* T} \boldsymbol{\theta}^{*}<-\frac{\sigma_{c}}{2 \lambda_{\max }\left(\Gamma^{-1}\right)} \tilde{\boldsymbol{\theta}}^{T} \Gamma^{-1} \tilde{\boldsymbol{\theta}}+\frac{\sigma_{c}}{2} \boldsymbol{\theta}^{* T} \boldsymbol{\theta}^{*}, \\
\Pi_{3}^{T_{g}} & <-\frac{\sigma_{f_{1}}}{2} \tilde{f}_{1}^{2}+\frac{\sigma_{f_{1}}}{2} \bar{f}_{1}^{2}+\rho_{\dot{f}_{1}} \rho_{\tilde{f}_{1}}, \quad \Pi_{4}^{T_{g}}<-\frac{\sigma_{\tilde{f}_{1}}}{2} \tilde{\rho}_{\tilde{f}_{1}}^{2}+\frac{\sigma_{\tilde{f}_{1}}}{2} \rho_{\tilde{f}_{1}}^{2},
\end{aligned}
$$

where $\lambda_{\max }\left(\Gamma^{-1}\right)$ is the maximum eigenvalue of $\Gamma^{-1}$. Finally, when considering (17), (30), and (31), it can be shown that 


$$
\dot{V}^{T_{g}}<-b_{1}^{T_{g}} V^{T_{g}}+b_{2}^{T_{g}}
$$

where $\quad 0<b_{1}^{T_{g}}=\min \left[2 k_{1, \omega_{g}}, 2 k_{2, \omega_{g}}, \sigma_{\tilde{f}_{1}}, \sigma_{f_{1}}, \sigma_{d_{1}}, \sigma_{c} / \lambda_{\max }\left(\Gamma^{-1}\right), 2 \Upsilon_{1}\right] \quad$ and $\quad 0<b_{2}^{T_{g}}=\eta_{d_{1}} D$ $+\frac{\sigma_{d_{1}}}{2} D^{2}+\frac{\sigma_{c}}{2} \boldsymbol{\theta}^{* 2}+\frac{\sigma_{f_{1}}}{2}{\overline{f_{1}}}^{2}+\rho_{\dot{f}_{1}} \rho_{\tilde{f}_{1}}+\frac{\sigma_{\tilde{f}_{1}}}{2} \rho_{\tilde{f}_{1}}{ }^{2}+\Upsilon_{2}$. Now, when considering Lemma 1 , it can be stated that $e_{\omega_{g}}$ and $\dot{e}_{\omega_{g}}$ are UUB.

In Fig. 6, the schematic diagram of the proposed generator torque controller is illustrated.

Note that, when considering (4) and (27), larger controller design parameters lead to higher drivetrain torsion angle and generator torque variation, which can be seen as negative effects. So, the design parameters are selected in such a way to keep the drivetrain torsion angle and generator torque variation at a reasonable level compared with the ICGC values.

When considering $f_{1}=b_{3} \Delta f_{G C}$, and the estimated fault, $\hat{f}_{1}$, the generator bias can be obtained as

$$
\hat{f}_{T_{g}}=\frac{\hat{f}_{1}}{b_{3} a_{g}},
$$

where $\hat{f}_{T_{g}}$ is the estimated generator torque bias. Indeed, the benefit of the proposed controller is that the fault effect is handled and no controller reconfiguration is needed. However, the estimated fault signal is generated, which can be used for many purposes, such as supervisory condition monitoring schemes as well as in the manual maintenance procedures.

\section{Pitch actuator controller design}

The pitch controller is designed in this section to fulfill the next control objective, i.e., keeping the pitch angle close to zero, despite the presence of pitch actuator bias. Additionally, an estimation of pitch actuator dynamic change is obtained, which can be implemented on the well-known baseline controller in the full load region ${ }^{2,7,25}$ to remove fault effects as the extension of the current study. The pitch tracking error is constructed as $e_{\beta}=\beta_{d}-\beta_{s}$. When considering (6), the pitch actuator dynamic behavior can be rewritten as

$$
\ddot{\beta}=-\omega_{n, N}^{2} \beta-2 \omega_{n, N} \xi_{N} \dot{\beta}+\omega_{n, N}^{2} \beta_{r e f}+f_{2}+d_{2},
$$

where $d_{2}=-\omega_{n, N}^{2} \nu_{P s}-2 \omega_{n, N} \xi_{N} \nu_{\dot{\beta}}$ and $f_{2}=\Delta \tilde{f}_{P A D}-\omega_{n, N}^{2} f_{P s} . \beta_{d}$ is the desired pitch angle, which is zero in a partial load operation. Also, $\beta_{d}$ is variable and should be tuned in the full load region, in which a simple industrial baseline controller can be used. ${ }^{2,19}$

Assumption 3. The accumulative fault, $f_{2}$, is assumed to be bounded, such that $\left|f_{2}\right| \leq \bar{f}_{2}$, where $\bar{f}_{2} \in \mathbb{R}^{+}$is an unknown constant. In fact, $f_{P S}$ is considered as a constant or a slowly varying additive step applied at the unknown fault time, i.e., $t_{f_{P S}}$. However, $\Delta \tilde{f}_{P A D}$, with respect

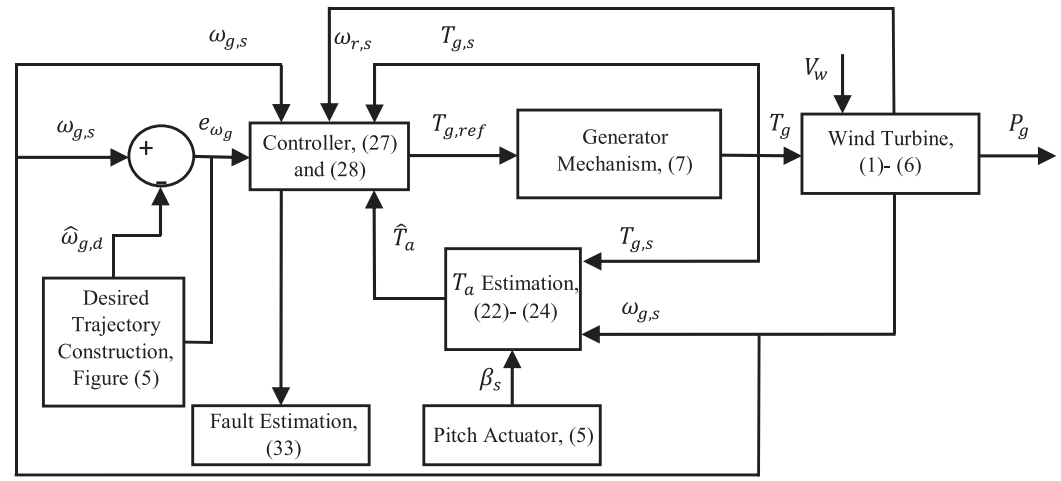

FIG. 6. Generator torque controller diagram. 
to limited actuator input and achievable states, is bounded. ${ }^{32}$ Also, the time derivative of the fault is assumed to be bounded, i.e., $\left|\dot{f}_{2}\right| \leq \rho_{\dot{f}_{2}},{ }^{2}$, where $\rho_{\dot{f}_{2}} \in \mathbb{R}^{+}$is unknown, and with the accumulative disturbance, $d_{2}$, it is assumed that $\left|d_{2}\right| \leq \bar{d}_{2}$, where $\bar{d}_{2}$ is an unknown constant. ${ }^{12}$

Now, to design the pitch actuator controller, a positive definite Lyapunov function is chosen as

$$
V_{1}^{\beta}=\frac{1}{2} e_{\beta}^{2},
$$

where its time derivative can be written as $\dot{V}_{1}^{\beta}=e_{\beta} \dot{e}_{\beta}$. Define a variable transformation as $e_{2, \beta}=\alpha_{\beta}-\dot{\beta}_{s}$, where $\alpha_{\beta}$ is the virtual control signal, which is chosen as

$$
\alpha_{\beta}=\dot{\beta}_{d}+k_{1, \beta} e_{\beta},
$$

where $k_{1, \beta}$ is a positive constant design parameter. Now, the pitch actuator controller is designed as

$$
\beta_{r e f}=\frac{1}{\omega_{n, N^{2}}}\left(g_{2}-\hat{f}_{2}+\frac{e_{2, \beta}}{\sqrt{e_{2, \beta}^{2}+\eta_{d_{2}}^{2}}} \hat{d}_{2}+k_{2, \beta} e_{2, \beta}\right),
$$

where $\eta_{d_{2}}$ and $k_{2, \beta}$ are positive constant design parameters, and $\hat{f}_{2}$ and $\hat{d}_{2}$ are estimations of $f_{2}$ and $d_{2}$, respectively. Also, $g_{2}=e_{\beta}+\dot{\alpha}_{\beta}+\omega_{n, N}^{2} \beta_{s}+2 \omega_{n, N} \xi_{N} \dot{\beta}_{s}$ and the adaptive laws are defined as

$$
\dot{\hat{d}}_{2}=\frac{e_{2, \beta}^{2}}{\sqrt{e_{2, \beta}^{2}+\eta_{d_{2}}^{2}}}-\sigma_{d_{2}} \hat{d}_{2}, \quad \dot{\hat{f}}_{2}=-e_{2, \beta}-\sigma_{f_{2}} \hat{f}_{2}, \quad \dot{\hat{\rho}}_{\tilde{f}_{2}}=-\sigma_{\tilde{f}_{2}} \hat{\rho}_{\tilde{f}_{2}},
$$

where $\sigma_{d_{2}}, \sigma_{f_{2}}$, and $\sigma_{\tilde{f}_{2}}$ are positive constant design parameters. The estimation errors are defined as $\tilde{d}_{2}=\hat{d}_{2}-\bar{d}_{2}$ and $\tilde{f}_{2}=\hat{f}_{2}-f_{2}$, and it is assumed that $\left|\tilde{f}_{2}\right|<\rho_{\tilde{f}_{2}}$, with unknown $\rho_{\tilde{f}_{2}} \in \mathbb{R}^{+}$. Estimation of $\rho_{\tilde{f}_{2}}$ is defined as $\hat{\rho}_{\tilde{f}_{2}}$ and its estimation error as $\tilde{\rho}_{\tilde{f}_{2}}=\hat{\rho}_{\tilde{f}_{2}}-\rho_{\tilde{f}_{2}}$.

Theorem 3. Consider the pitch actuator (6) and proposed pitch controller (37). Let the initial conditions of the pitch actuator be bounded. Then, for all generator torque, $e_{\beta}$ and $\dot{e}_{\beta}$ are UUB.

Proof. Choose a positive definite Lyapunov function, as

$$
V^{\beta}=V_{1}^{\beta}+\frac{1}{2} e_{2, \beta}^{2}+\frac{1}{2} \tilde{d}_{2}^{2}+\frac{1}{2} \tilde{f}_{2}^{2}+\frac{1}{2} \tilde{\rho}_{\tilde{f}_{2}}^{2} .
$$

When taking the time derivative of (39) obtains, $\dot{V}^{\beta}=\dot{V}_{1}^{\beta}+e_{2, \beta} \dot{e}_{2, \beta}+\tilde{d}_{2} \dot{\tilde{d}}_{2}+\tilde{f}_{2} \dot{\tilde{f}}_{2}+\tilde{\rho}_{\tilde{f}_{2}} \dot{\tilde{\rho}}_{\tilde{f}_{2}}$, which can be rewritten as

$$
\dot{V}^{\beta}=\sum_{i=1}^{3} \Pi_{i}^{\beta}-k_{1, \beta} e_{\beta}^{2}-k_{2, \beta} e_{2, \beta}^{2},
$$

where $\quad \Pi_{1}^{\beta}=e_{2, \beta}\left(\hat{f}_{2}-f_{2}-\tilde{f}_{2}\right)-\sigma_{f_{2}} \hat{f}_{2} \tilde{f}_{2}-\dot{f}_{2} \tilde{f}_{2}, \quad \Pi_{2}^{\beta}=-e_{2, \beta} d_{2}+e_{2, \beta}^{2}\left(\tilde{d}_{2}-\hat{d}_{2}\right) / \sqrt{e_{2, \beta}^{2}+\eta_{d_{2}}^{2}}$ $-\sigma_{d_{2}} \hat{d}_{2} \tilde{d}_{2}$ and $\Pi_{3}^{\beta}=-\sigma_{\tilde{f}_{2}} \hat{\rho}_{\tilde{f}_{2}} \tilde{\rho}_{\tilde{f}_{2}}$. According to Lemmas 2 and 3, it can be shown that

$$
\begin{aligned}
& \Pi_{1}^{\beta}<-\frac{\sigma_{f_{2}}}{2} \tilde{f}_{2}^{2}+\frac{\sigma_{f_{2}}}{2} \bar{f}_{2}^{2}+\rho_{\dot{f}_{2}} \rho_{\tilde{f}_{2}}, \\
& \Pi_{2}^{\beta}<\bar{d}_{2}\left(\left|e_{2, \beta}\right|-\frac{e_{2, \beta}^{2}}{\sqrt{e_{2, \beta}^{2}+\eta_{d_{2}}^{2}}}\right)-\sigma_{d_{2}} \hat{d}_{2} \tilde{d}_{2}<\bar{d}_{2} \eta_{d_{2}}-\frac{\sigma_{d_{2}}}{2} \tilde{d}_{2}^{2}+\frac{\sigma_{d_{2}}}{2} \bar{d}_{2}^{2} \\
& \Pi_{3}^{\beta}<-\frac{\sigma_{\tilde{f}_{2}}}{2} \tilde{\rho}_{\tilde{f}_{2}}{ }^{2}+\frac{\sigma_{\tilde{f}_{2}}}{2} \rho_{\tilde{f}_{2}}^{2} .
\end{aligned}
$$


Accordingly, when considering (40) and (41), it can be shown that

$$
\dot{V}^{\beta}<-b_{1}^{\beta} V^{\beta}+b_{2}^{\beta}
$$

where $0<b_{1}^{\beta}=\min \left(2 k_{1, \beta}, 2 k_{2, \beta}, \sigma_{\tilde{f}_{2}}, \sigma_{f_{2}}, \sigma_{d_{2}}\right) \quad$ and $\quad 0<b_{2}^{\beta}=\frac{\sigma_{f_{2}}}{2} \bar{f}_{2}^{2}+\rho_{\tilde{f}_{2}} \rho_{\tilde{f}_{2}}+\bar{d}_{2} \eta_{d_{2}}+\frac{\sigma_{d_{2}}}{2} \bar{d}_{2}^{2}$ $+\frac{\sigma_{\tilde{f}_{2}}}{2} \rho_{\tilde{f}_{2}}{ }^{2}$. Now, when considering Lemma 1, it can be stated that $e_{\beta}$ and $\dot{e}_{\beta}$ are UUB.

Now, the pitch actuator bias and dynamic change are going to be approximated. The auxiliary signals for different cases of pitch actuator dynamic change are calculated and compared with the estimated fault $\hat{f}_{2}$ to detect and isolate dynamic change. When considering (6) and Table I, the auxiliary signals are calculated as

$$
\begin{aligned}
f_{\text {auxiliary,faultfree }} & =0 \Delta\left(\tilde{\omega}_{n}{ }^{2}\right) \beta_{s}-0 \Delta\left(\tilde{\omega}_{n} \tilde{\xi}\right) \dot{\beta}_{s}+0 \Delta\left(\tilde{\omega}_{n}{ }^{2}\right) \beta_{\text {ref }}=0 \\
f_{\text {auxiliary, pump wear }} & =-0.6316 \Delta\left(\tilde{\omega}_{n}{ }^{2}\right) \beta_{s}-0.59376 \Delta\left(\tilde{\omega}_{n} \tilde{\xi}\right) \dot{\beta}_{s}+0.6316 \Delta\left(\tilde{\omega}_{n}{ }^{2}\right) \beta_{\text {ref }}, \\
f_{\text {auxiliary, hydraulic leak }} & =-\Delta\left(\tilde{\omega}_{n}{ }^{2}\right) \beta_{s}-1.75706 \Delta\left(\tilde{\omega}_{n} \tilde{\xi}\right) \dot{\beta}_{s}+\Delta\left(\tilde{\omega}_{n}{ }^{2}\right) \beta_{\text {ref }} \\
f_{\text {auxiliary, highair }} & =-0.81083 \Delta\left(\tilde{\omega}_{n}^{2}\right) \beta_{s}-2 \Delta\left(\tilde{\omega}_{n} \tilde{\xi}\right) \dot{\beta}_{s}+0.81083 \Delta\left(\tilde{\omega}_{n}^{2}\right) \beta_{r e f}
\end{aligned}
$$

where, in $f_{\text {auxiliary }, X}, X$ represents the dynamic change type and is replaced with either faultfree, pump wear, hydraulic leak, or high air. Also, $\beta_{s}$ and $\dot{\beta}_{s}$ are measured pitch angle and its rate, respectively, and $\beta_{\text {ref }}$ is defined in (37). The other parameters are defined in (6). To proceed with fault estimation of the pitch actuator, first, the dynamic change case is indicated and it is assumed that there is no pitch actuator bias. When considering $f_{2}=\Delta \tilde{f}_{P A D}-\omega_{n, N}^{2} f_{P S}$, in the absence of $f_{P S}$, it can be obtained that $f_{2}=\Delta \tilde{f}_{P A D}$. So, by using all auxiliary signals $f_{\text {auxiliary }, X}$, as (43), the most similar one to the estimated accumulative fault, $\hat{f}_{2}$, is selected as the dynamic change case. So, similarity indices are utilized, on which basis, the fault isolation is conducted. The root-mean-squared-error (RMSE) and the variance-accounted-for (VAF) indices are used in this paper, which are defined as

$$
\begin{aligned}
\operatorname{RMSE}_{X} & =\sqrt{\frac{1}{T} \int_{0}^{T_{\text {exe }}}\left(f_{\text {auxiliary }, X}-\hat{f}_{2}\right)^{2} d t}, \\
V A F_{X} & =\left[1-\frac{\operatorname{var}\left(f_{\text {auxiliary }, X}-\hat{f}_{2}\right)}{\operatorname{var}\left(f_{\text {auxiliary }, X}\right)}\right] \times 100 \%,
\end{aligned}
$$

where $X$ represents the dynamic change type and $T_{\text {exe }}$ is the execution time. In the ideal fault detection case, the RMSE and VAF should be zero and 100\%, respectively. Indeed, when using (44), the case $X$, which leads to the RMSE and VAF indices close to zero and $100 \%$, respectively, is selected as the corresponding dynamic change case, i.e., $\hat{X}$. It should be noted that pitch bias, $f_{P S}$, is considered as an added constant on the accumulative fault. So, even in the presence of pitch bias with dynamic change, only the RMSE index deviates significantly from zero, but the VAF index still properly indicates the correct dynamic change. Now, after indicating $\hat{X}$, the pitch actuator bias is estimated. When considering $f_{2}=f_{\text {auxiliary }, \hat{X}}-\omega_{n, N}^{2} f_{P S}$, where $f_{\text {auxiliary }, \hat{X}}$ is calculated auxiliary signal by using (43) for indicated dynamic change $\hat{X}$, the estimation of pitch actuator bias, $\hat{f}_{P_{s}}$, is as

$$
f_{P s}=\frac{f_{\text {auxiliary }, \hat{X}}-\hat{f}_{2}}{\omega_{n, N^{2}}} .
$$

\section{SIMULATION}

In this section, the proposed controllers, (27) and (37), are evaluated and the results are compared with the ICGC values. The satisfying behaviour of the wind turbine when using the 


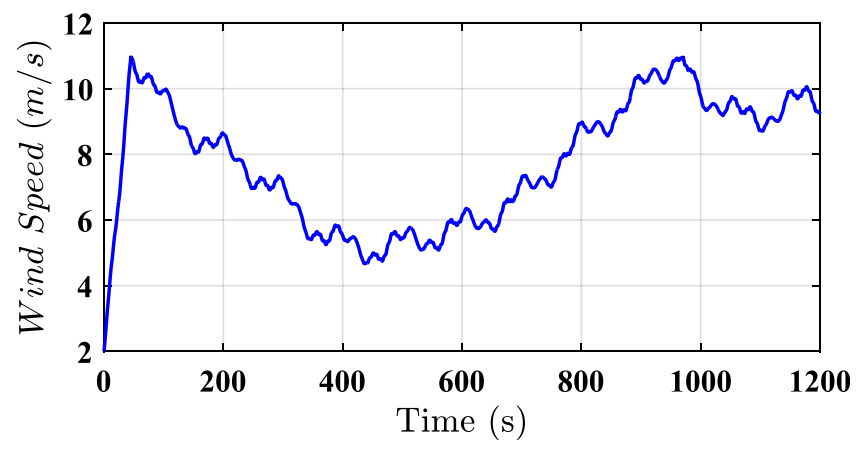

FIG. 7. Wind speed.

ICGC may lead to model verification, ${ }^{6}$ although, this is not the main reason for using the ICGC. It should be noted that more accurate desired trajectory tracking, when taking wind speed variation into account, leads to more $S$ and GTV. So, there should be a trade-off between Efficiency, S, and GTV; accordingly, in this paper, it is expected to increase Efficiency while keeping $S$ and $G T V$ at a reasonable level compared with the ICGC values, which has industrial acceptability. First, the fault free situation is studied by using both the proposed controller and the ICGC via considering control criteria. Then, the fault detection of the proposed controller for pitch mechanism is evaluated, and, also, its fault tolerant capability is considered. The generator controller response, including fault detection and fault effect removal, is studied. The effectiveness of the proposed controller for a real wind speed profile is evaluated. Finally, the simulation results are discussed. It should be noted that all numerical simulations were conducted by using MATLAB/Simulink software.

\section{A. Fault-free situation}

The simulation results of the generated power augmented with the ICGC and the proposed controller, by using wind speed shown in Fig. $7^{6,20}$ are shown in Fig. 8. It is assumed that there is no dynamic change or pitch bias in the pitch mechanisms and no torque bias in the generator. Also, power coefficients that use both controllers are shown in Fig. 9. The initial response using the proposed controller is faster than the initial response using ICGC. When considering the effect of this variation on the wind turbine, the induced drivetrain torsion angle, $\dot{\theta}_{\Delta}$, is shown in Fig. 10, for both controllers. It is obvious that the induced gearbox torsion angle for both controllers is in the same order. It also confirms the assumption that was made in keeping $\dot{\theta}_{\Delta}$ as close to zero as possible, in the generator torque control design. In Fig. 8, at some times, where the wind speed reduces after a high value, the generated power is more than the optimal power, because the stored kinetic energy is being converted to electrical energy. ${ }^{20}$ The tracking error and generator torque for both controllers are shown in Figs. 11 and 12, respectively.

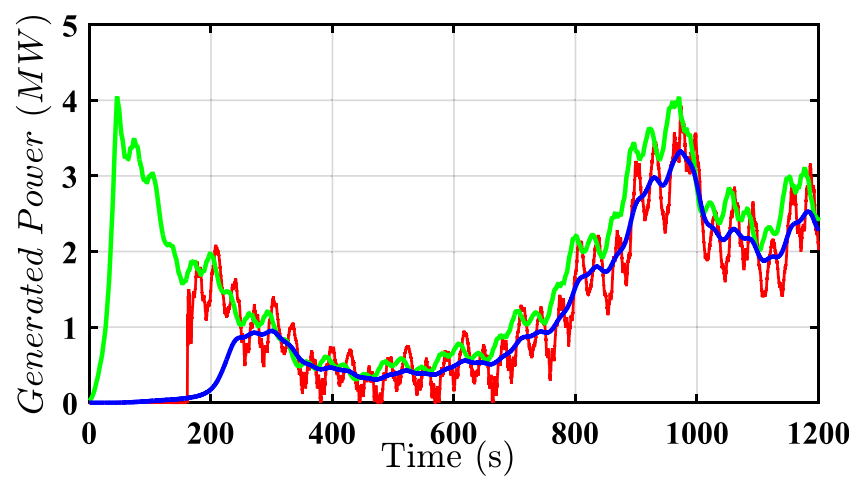

FIG. 8. Generated power by using the proposed controller (red line), the ICGC (blue line), and optimal power (green line). 


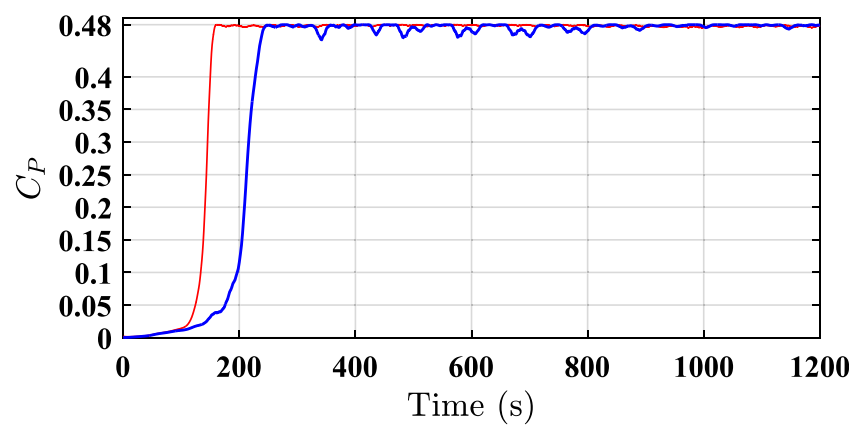

FIG. 9. Power coefficient by using the proposed controller (red line) and the ICGC (blue line) in fault free situation.

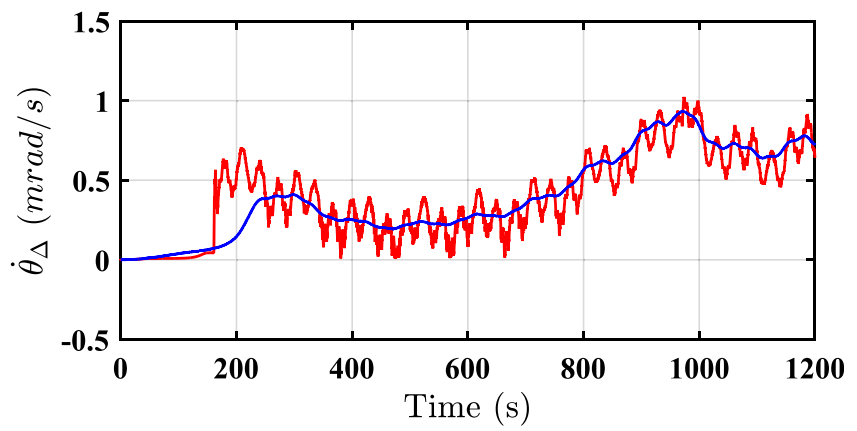

FIG. 10. Torsion angle of drive train by using the proposed controller (red line) and the ICGC (blue line).

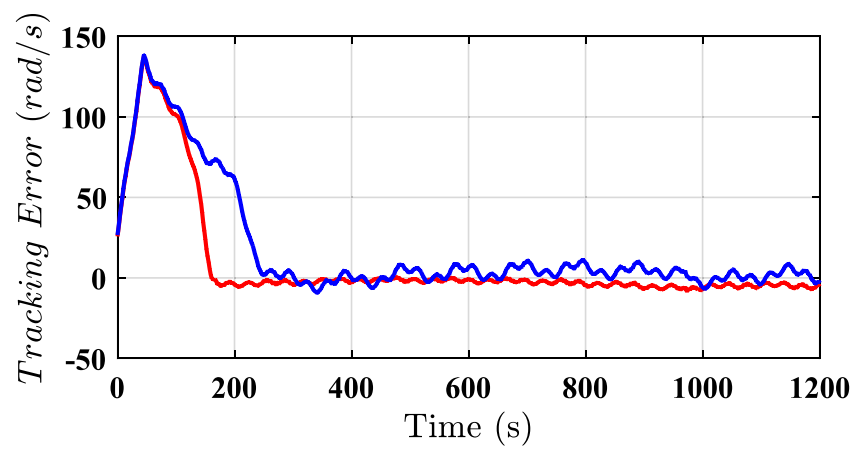

FIG. 11. Tracking error by using the proposed controller (red line) and ICGC (blue line).

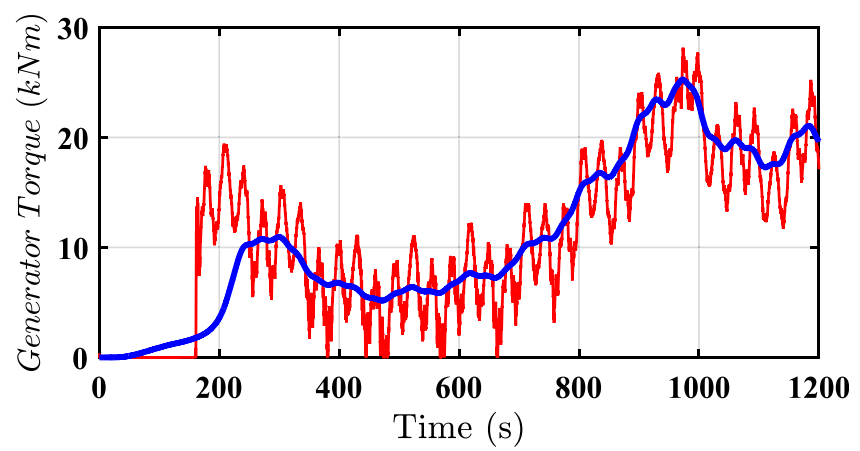

FIG. 12. Generator torque by using the proposed controller (red line) and the ICGC (blue line). in fault free situation. 


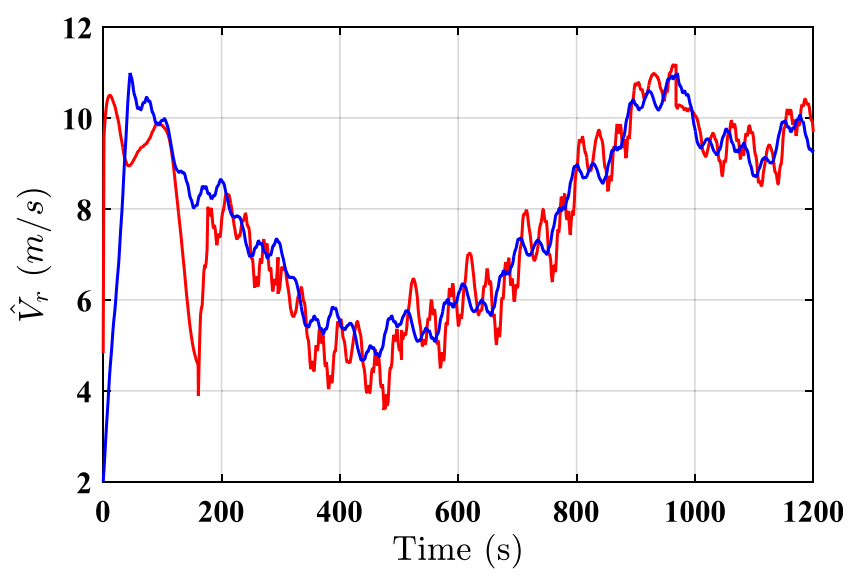

FIG. 13. Estimated (red line) and actual (blue line) wind speed.

Considering the ICGC results, including Figs. 8 and 9, can lead to model verification. Finally, the estimated wind speed is calculated by using (9) and (11), which is shown in Fig. 13. A high variation of produced power by using the proposed controller, as illustrated in Fig. 8, is due to the desired trajectory construction and the aerodynamic torque estimation based on RBFNN. Indeed, when considering Fig. 13, the fluctuation of the estimated wind speed around the actual one is obvious. The desired trajectory has been constructed when using (11), in which radial basis functions form the basis functions. Accordingly, when using (9), the wind speed is estimated. Both the constructed desired trajectory and estimated aerodynamic torque have been utilized in the generator torque controller, see (27). Therefore, the fluctuation of the constructed desired trajectory and estimated aerodynamic torque, led to high variation of generator torque and, consequently, when considering the produced power, i.e., $P_{a}=\eta_{g} T_{g} \omega_{g}$, causes a high variation of power. So, reconsidering Fig. 8, the produced power has fluctuated in the same manner as the estimated wind speed. To compare the results of the two controllers, the values of the control criteria are compared in Table II. It can be seen that more power is extracted by using the proposed controller compared with the reference controller, although, this is not the main purpose of the proposed controller, which is its FTC capabilities, considered in Sec. VB.

\section{B. Fault tolerant control}

The FTC capabilities of the proposed controllers are evaluated. Also, the faults are augmented in the ICGC scheme to show the effect of the considered faults, and the results are compared with those from the proposed controller.

\section{Pitch actuator fault detection}

In this section, the fault detection of the pitch actuator system is considered by using the proposed controller (36). In this regard, first, the dynamic change of the pitch actuator is considered. To avoid a trivial response, an abrupt nonzero time varying input is applied to the pitch system for a small time interval to study its behaviour. For $6 \mathrm{~s}$, a sinusoidal wave is used as $\beta_{d}=1 \sin (t){ }^{3}$ The pitch sensor output for the fault-free case, pump wear, high air content, and

TABLE II. Values of the control criteria for fault-free situation.

\begin{tabular}{lcccccc}
\hline \hline Fault free situation & $\mathrm{E}(\mathrm{GJ})$ & Efficiency & $S\left(\mu \mathrm{rad}^{2} / 2\right)$ & $G T V(\mathrm{MNm})$ & $\operatorname{Max}\left(T_{g}\right)(\mathrm{kNm})$ & $\mathrm{STD}\left(T_{g}\right)(\mathrm{kNm})$ \\
\hline Proposed controller & 1.486 & $70 \%$ & 46.7 & 17.88 & 28.10 & 7.32 \\
Reference controller & 1.394 & $65 \%$ & 0.6154 & 0.3694 & 25.30 & 7.27 \\
\hline \hline
\end{tabular}


hydraulic leak cases are shown in Fig. 14(a). The pitch actuator response is very similar in all situations, which is the case in Fig. 3, where the time delay for each case changes by $0.5 \mathrm{~s}$ at the most. Also, in each case, the estimated fault and calculated auxiliary signals are shown in Figs. 14(b) and 14(c), respectively, on which basis the detection indices, (44), are calculated and shown in Table III. According to Table III, for each fault case, when using the estimated accumulated fault, $\hat{f}_{2}$, and comparing the indices for each auxiliary signal, it is expected that RMSE and VAF values would be close to zero and 100, respectively. Therefore, when comparing all calculated indices, the auxiliary signal, which leads to the smallest RMSE and closest VAF to 100 , is selected as the detected dynamic change case of the pitch actuator. In the last column of Table III, the detected fault is represented, which shows that the proposed fault detection accurately detects all pitch actuator dynamic change cases.

To evaluate the detection of pitch actuator bias, $f_{P_{S}}=10^{\circ}$, is added to the pitch dynamic response and by using (44), the estimated pitch bias, $\hat{f}_{P_{S}}$, is shown in Fig. 15. The reference pitch angle is set to $\beta_{r e f}=1 \sin (t),{ }^{3}$ the same as the dynamic change detection. In Fig. 15, $\hat{f}_{P_{s}}$ is accurately estimated.

\section{Pitch actuator fault tolerant control}

First, the proposed pitch actuator controller, (37), is evaluated, where the dynamic changes due to pump wear, hydraulic leak, and high air content in the oil are introduced. Also, the pitch actuator bias is added to the pitch mechanism. In addition, sensor measurement is contaminated with noise. Accordingly, to only consider the dynamic change, sensor noise and pitch bias are removed, which will be considered afterward. Indeed, the sensor noise and pitch bias have more effect on pitch angle than the dynamic change, which reduces the visibility of the dynamic change. Actually, removing sensor noise and bias is just for the sake of consideration. Also, $\beta_{d}=0^{\circ}$. The initial pitch angle is selected as $5^{\circ}$ to let the delay in response be distinguishable. The results are illustrated in Fig. 16. By using the proposed pitch controller reduces the delays due to the induced dynamic change significantly, where the response is even better than the faultfree case with no controller.

To consider all types of faults altogether, pitch sensor noise, $\nu_{P s}$, and pitch bias, $f_{P s}$, i.e., $10^{\circ}$, are introduced to the model, and it is expected that the pitch bias is removed, and,
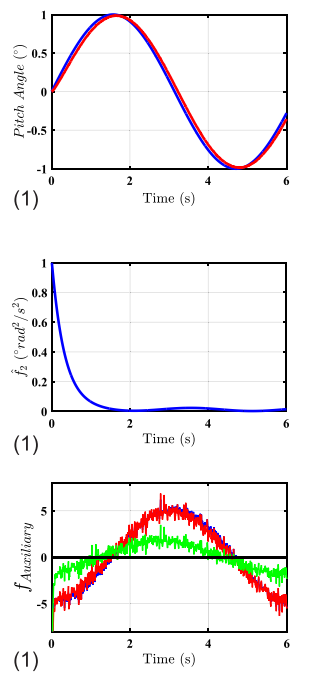
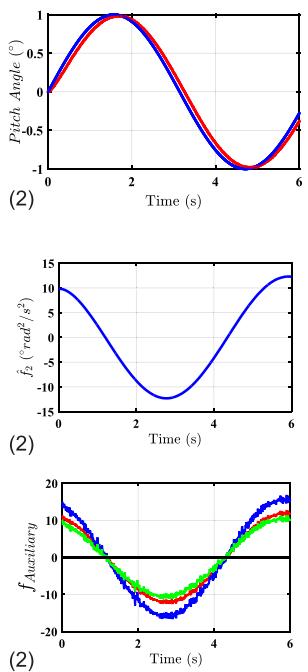

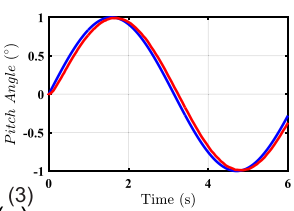

(a)
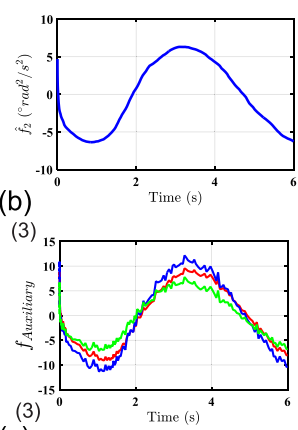

(c)
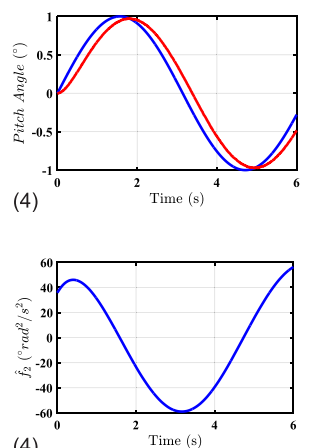

(4)

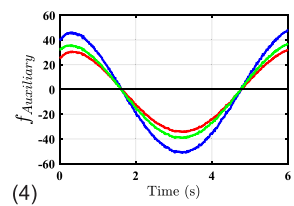

FIG. 14. Fault detection in pitch actuator due to dynamic change. (a) Reference pitch (blue line) and pitch sensor output (red line). (1) Fault-free case, (2) pump wear case, (3) high air content case, and (4) hydraulic leak case. (b) Estimated fault signal. (1) Fault-free case, (2) pump wear case, (3) high air content case, and (4) hydraulic leak case. (c) Calculated auxiliary signal for fault-free (black), pump wear (red), hydraulic leak (blue), and high air content (green). (1) Fault-free case, (2) pump wear case, (3) high air content case, and (4) hydraulic leak case. 
TABLE III. Pitch actuator dynamic change fault detection indices. (Text in bold indicates that the cell is chosen).

\begin{tabular}{|c|c|c|c|c|c|c|c|c|c|}
\hline \multirow[b]{3}{*}{ Fault free } & \multicolumn{8}{|c|}{ Calculated auxiliary signal } & \multirow{3}{*}{$\begin{array}{l}\text { Decision } \\
\text { No fault }\end{array}$} \\
\hline & \multicolumn{2}{|c|}{ Fault free } & \multicolumn{2}{|c|}{ Pump wear } & \multicolumn{2}{|c|}{ Hydraulic leak } & \multicolumn{2}{|c|}{ High air content } & \\
\hline & RMSE & VAF & RMSE & VAF & RMSE & VAF & RMSE & VAF & \\
\hline & 0.136 & 95.3 & 3.887 & 2.71 & 3.918 & 3.010 & 1.838 & 7.784 & \\
\hline \multirow[t]{2}{*}{ Pump wear } & RMSE & VAF & RMSE & VAF & RMSE & VAF & RMSE & VAF & Pump wear is selected. \\
\hline & 8.400 & 0.82 & 0.469 & 99.69 & 2.585 & 94.353 & 1.386 & 96.415 & \\
\hline \multirow[t]{2}{*}{ Hydraulic leak } & RMSE & VAF & RMSE & VAF & RMSE & VAF & RMSE & VAF & Hydraulic leak is selected. \\
\hline & 40.85 & 0.05 & 17.003 & 50.026 & 5.606 & 97.647 & 13.513 & 76.046 & \\
\hline \multirow[t]{2}{*}{ High air content } & RMSE & VAF & RMSE & VAF & RMSE & VAF & RMSE & VAF & High air content is selected. \\
\hline & 56.811 & 0.08 & 2.2478 & 86.176 & 3.349 & 79.648 & 0.780 & 97.128 & \\
\hline
\end{tabular}

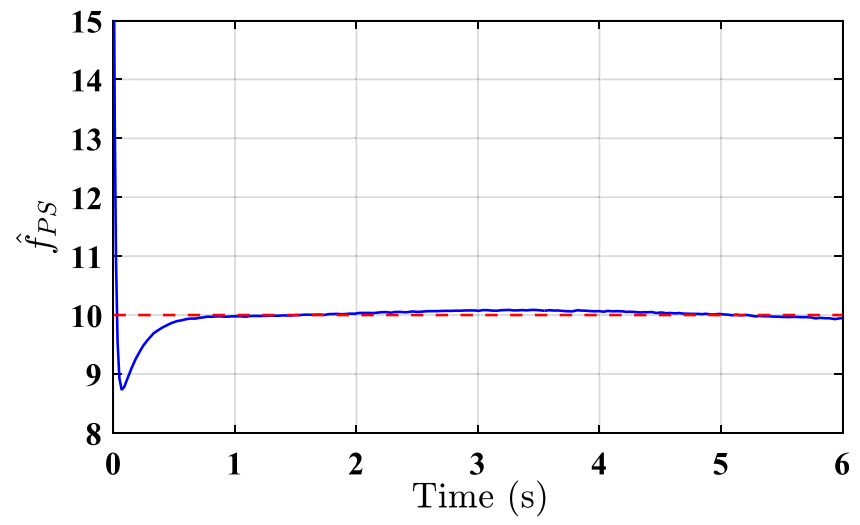

FIG. 15. The estimated pitch actuator bias (blue line) and the actual one (red line).

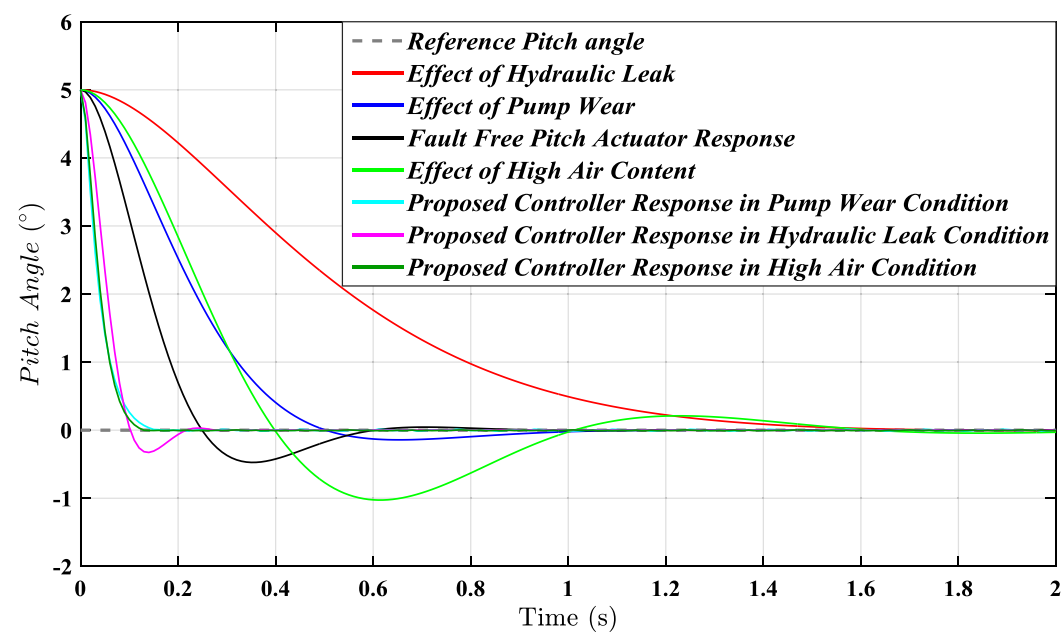

FIG. 16. Pitch actuator controller response without sensor bias and noise, fault free with no controller (black line), pump wear condition (blue line), hydraulic leak condition (red line), high air condition (light green line), pump wear with controller (light blue line), hydraulic leak with controller (pink line), and high air in the oil with controller (dark green). 

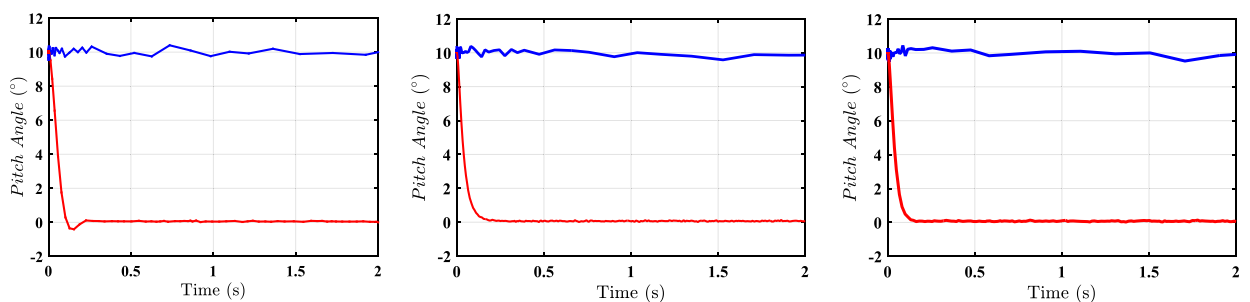

FIG. 17. Pitch actuator controller response (red line) and without controller (blue line) in (a) hydraulic leak, (b) pump wear, and (c) high air content conditions with sensor noise and pitch bias.

meanwhile, the noise should not cause instability and should be reduced. In Fig. 17, the proposed pitch controller results, in the presence of $\nu_{P_{S}}$ and $f_{P_{S}}$, for pump wear, hydraulic leak, and high air situations, are illustrated, where the reference pitch angle is $0^{\circ}$. In each of the three cases, the pitch bias is removed. Also, when considering the dynamic change, it is obvious that the response of the pitch actuator by using the proposed controller is similar to the fault-free one, comparing Figs. 16 and 17. Finally, it can be stated that the proposed pitch controller is able to handle the pitch dynamic change and pitch bias. Although, in partial load operation, the pitch angle is enough to be set to $0^{\circ}$ by using the proposed controller, it is guaranteed that this aim is met by using the sensor measurement, which is contaminated with noise or else the pitch actuator may introduce the pitch bias. Also, this controller is readily capable of being used in full load operation, where the reference pitch angle is not fixed anymore and should be tuned to keep the generated power at its nominal power. Indeed, via the proposed controller, the results show that the pitch angle is guaranteed to track the reference one, whether it is fixed, i.e., in a partial load operation, which is of interest of this paper, or not, i.e., in full load operation, despite the presence of pitch bias, dynamic change, and pitch noise. Also, in full load operation by using the proposed controller, the dynamic change of the pitch actuator can be detected. However, the dynamic change, in the case of the zero degree fixed pitch angle, is not very effective, as shown in Fig. 16. So, in Sec. VB 3, only the pitch actuator bias is considered.

\section{Fault tolerant control of wind turbine by using both proposed generator and pitch controllers}

The generator torque controller, (27), alongside the pitch controller, (37), are applied to the wind turbine model, and it is expected that, despite the generator bias, i.e., $f_{T_{g}}$, pitch actuator bias, i.e., $f_{P S}$ and wind speed variation, which is shown in Fig. 7, the power coefficient is being kept at its maximum value, and, accordingly, the generated power tracks the maximum one. Also, the considered faults are applied to the ICGC to study the effects of faults on the wind turbine behavior. The fault scenario is summarized in Table IV. In this fault scenario, to study the effect of generator fault, which is more likely in partial load operation, ${ }^{16}$ two different generator biases are introduced. Also, to evaluate the fault tolerance capability accurately, a consecutive pitch bias is considered, which starts at $900 \mathrm{~s}$, exactly when the second generator bias ends.

Figures 18 and 19 show the power coefficient and generated power when using the proposed controller and the ICGC, respectively. It is obvious that the proposed controller keeps the power coefficient at its maximum one, whereas the reference control results are significantly

TABLE IV. First fault scenario.

\begin{tabular}{lcc}
\hline \hline Fault scenario 1 & Value & Period \\
\hline Generator bias & $f_{T_{g}}=2000 \mathrm{Nm}$ & $300 \mathrm{~s}-500 \mathrm{~s}$ \\
Generator bias & $f_{T_{g}}=5000 \mathrm{Nm}$ & $700 \mathrm{~s}-900 \mathrm{~s}$ \\
Pitch bias & $f_{P s}=10^{\circ}$ & $900 \mathrm{~s}-1100 \mathrm{~s}$ \\
\hline \hline
\end{tabular}




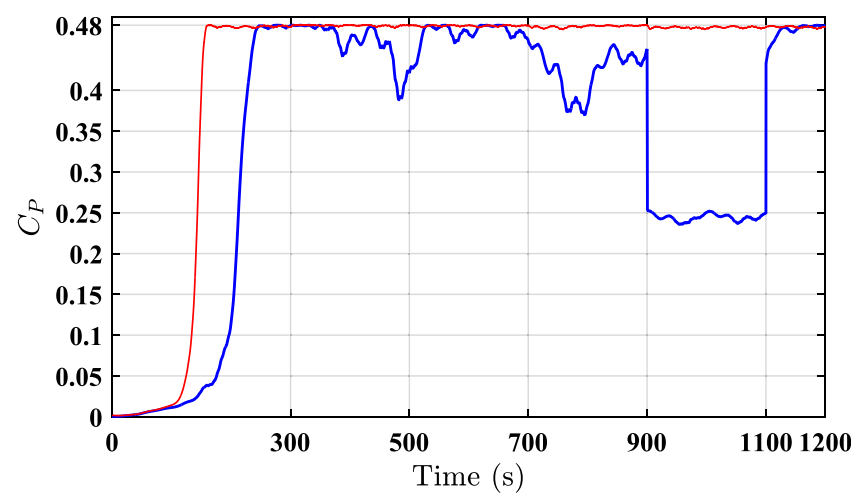

FIG. 18. Power coefficient by using the proposed controller (red line) and the ICGC (blue line).

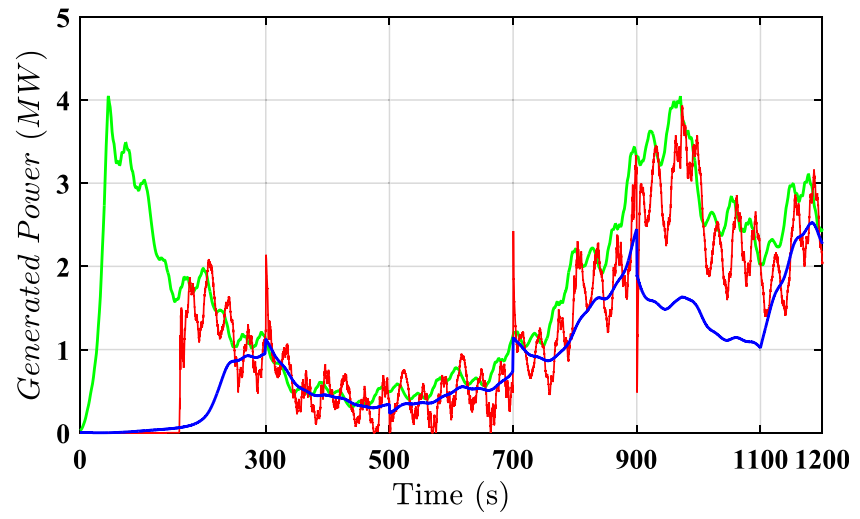

FIG. 19. Generated power by using the proposed controller (red line) and the ICGC (blue line) for first fault scenario and the optimal power (green line).

decreased in faulty periods. It is obvious that this power curve is similar to the fault-free one in Fig. 9, using the proposed controller. The tracking error, reference generator torque, reference pitch angle, and drivetrain torsion angle are illustrated in Figs. 20-23, respectively. It can be seen that the tracking error using the proposed controller is similar to the fault-free situation in Fig. 11. Also, the generator torque is kept at the same value unlike the corresponding result from the ICGC. At 300 s and 700 s, when the generator biases are applied, sudden deviations from the fault-free case are seen, after which their effects are completely removed by using the proposed controller. Also, the same result can be obtained when considering Fig. 22, in which

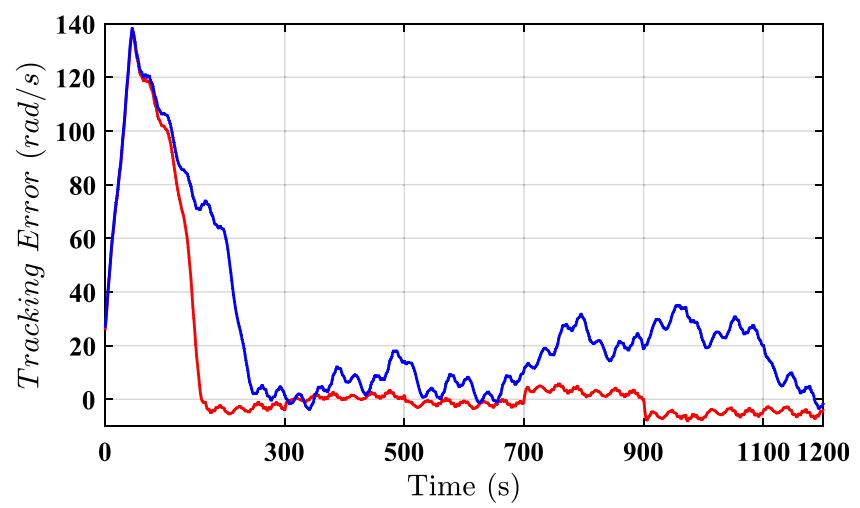

FIG. 20. Tracking error by using the proposed controller (red line) and the ICGC (blue line). 


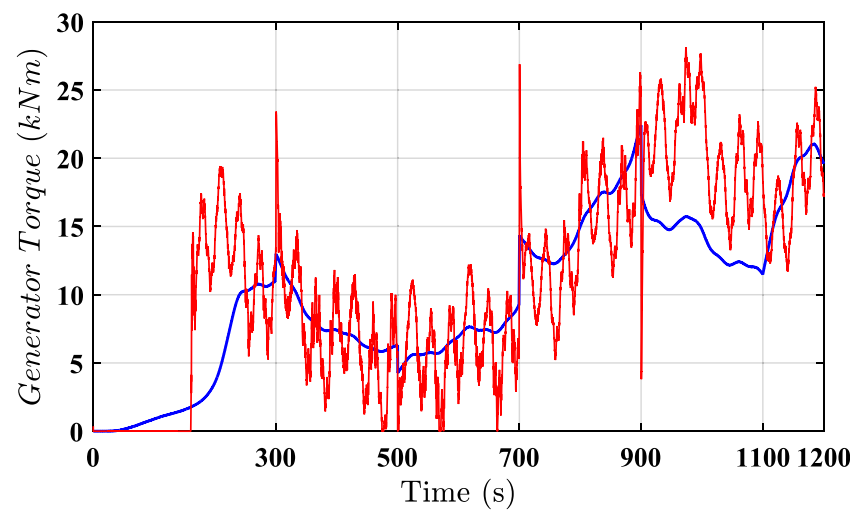

FIG. 21. Generator torque by using the proposed controller (red line) and the ICGC (blue line).

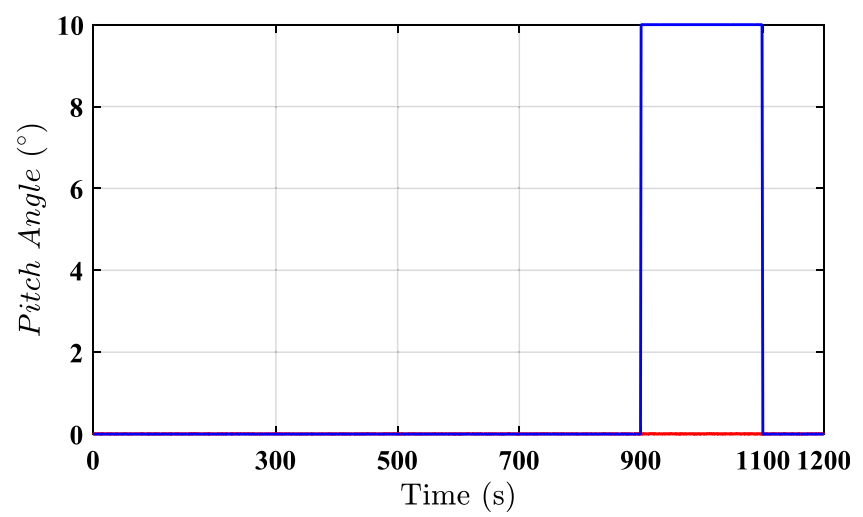

FIG. 22. Pitch angle by using the proposed controller (red line) and the ICGC (blue line).

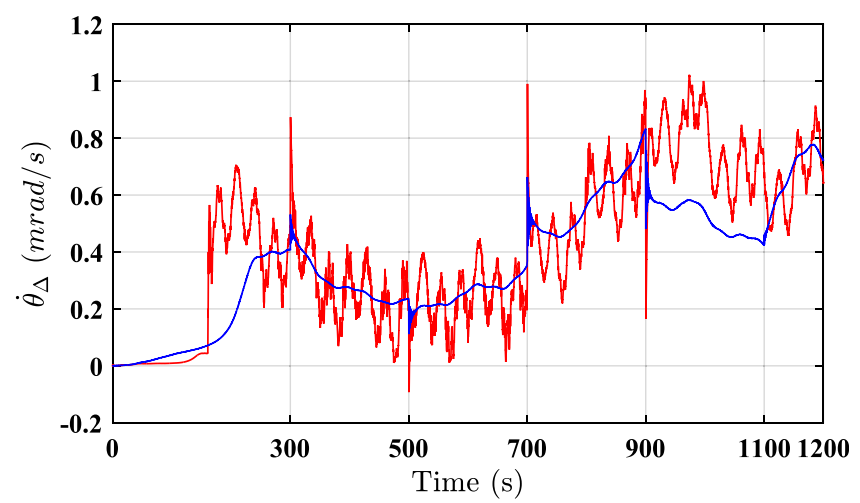

FIG. 23. Torsion angle of drivetrain by using the proposed controller (red line) and the ICGC (blue line).

by using the proposed pitch angle controller, the effect of pitch bias is removed. The estimated generator and pitch biases are depicted in Figs. 24 and 25, respectively. When considering the tracking error, the initial deviation of the estimated generator bias should not be taken into consideration because, at this time period, the desired trajectory tracking error has not yet been reduced, after which the generator biases are accurately estimated. However, because of the fast response of the pitch actuator, the pitch bias is estimated precisely over the whole simulation time. Also, the resulting control criteria are shown in Table V. It is obvious that the efficiency from using the proposed controller is kept the same as the fault-free one, while the 


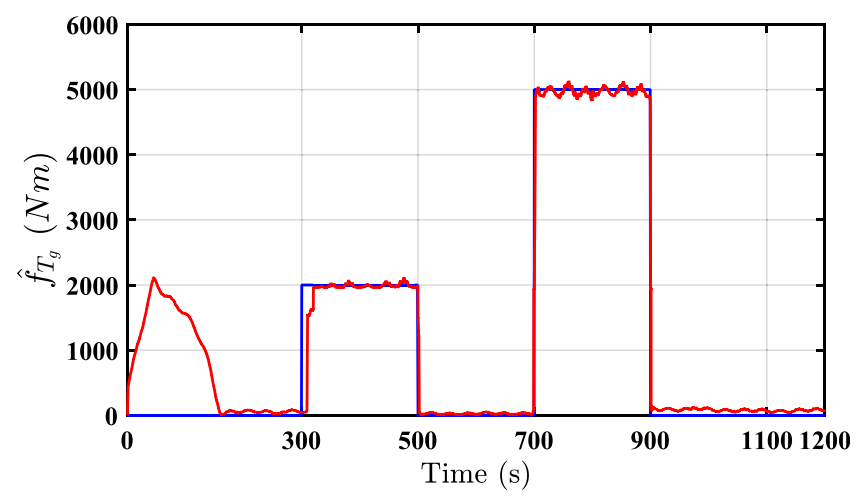

FIG. 24. Estimated (red line) and actual (blue line) generator bias.

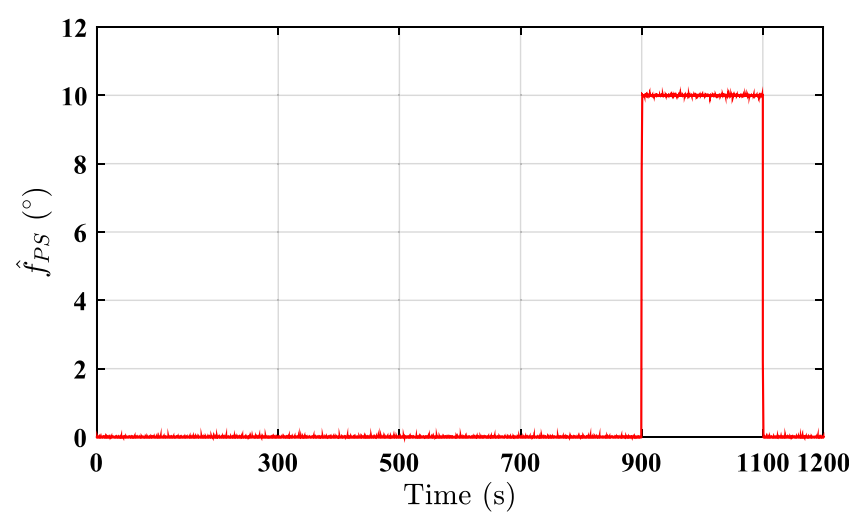

FIG. 25. Estimated (red line) and actual (blue line) pitch bias.

TABLE V. Values of the control criteria with the first-fault scenario.

\begin{tabular}{lcccccc}
\hline \hline First-fault scenario & $\mathrm{E}(\mathrm{GJ})$ & Efficiency & $S\left(\mu \mathrm{rad}^{2} / 2\right)$ & GTV $(\mathrm{MNm})$ & $\operatorname{Max}\left(T_{g}\right)(\mathrm{kNm})$ & $\mathrm{STD}\left(T_{g}\right)(\mathrm{kNm})$ \\
\hline Proposed controller & 1.483 & $69.5 \%$ & 68.8 & 18.49 & 28.14 & 7.38 \\
Reference controller & 1.1 & $52 \%$ & 32.55 & 0.3539 & 22.42 & 5.88 \\
\hline \hline
\end{tabular}

corresponding results when using the ICGC is decreased significantly. However, the induced drive train torsion stress, $S$, remains in the same order when using the proposed controller, but when considering the corresponding result by using the ICGC, this criterion is approximately 54 times larger than the fault free one. Also, when considering the other criteria, the advantage of the proposed controller can be concluded.

To further investigate the proposed controllers' performance, a realistic wind speed profile, as shown in Fig. 26, with a mean wind speed of $7.08 \mathrm{~m} / \mathrm{s},{ }^{20}$ is fed into the wind turbine, including the fault scenarios shown in Table VI. The control criteria are summarized in Table VII. The same results can be obtained when considering the performance of the proposed controller in both fault-free and faulty situations compared with the corresponding results when using the ICGC.

\section{Discussion}

In the simulations, for the fault-free case, the initial response from using the proposed controller has been improved, as shown in Figs. 8 and 9. Also, the fluctuations of generated power, induced torsion angle, and generator torque, can be seen in Figs. 8, 10, and 12, respectively. In 


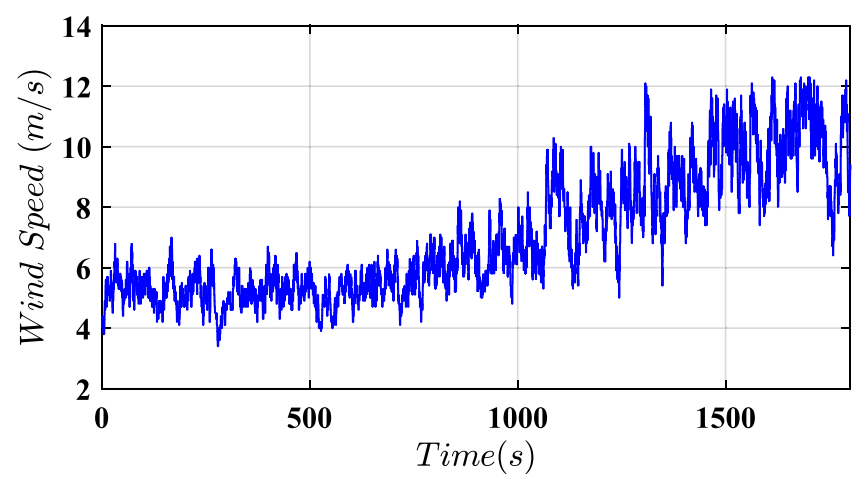

FIG. 26. Real wind speed profile.

TABLE VI. Second-fault scenario.

\begin{tabular}{lcc}
\hline \hline Fault scenario 2 & Value & Period \\
\hline Generator bias & $f_{T_{g}}=5000 \mathrm{Nm}$ & $100 \mathrm{~s}-300 \mathrm{~s}$ \\
Generator bias & $f_{T_{g}}=7000 \mathrm{Nm}$ & $900 \mathrm{~s}-1100 \mathrm{~s}$ \\
Pitch bias & $f_{P_{s}}=5^{\circ}$ & $500 \mathrm{~s}-700 \mathrm{~s}$ \\
Pitch bias & $f_{P_{s}}=10^{\circ}$ & $1300 \mathrm{~s}-1500 \mathrm{~s}$ \\
\hline \hline
\end{tabular}

TABLE VII. Values of the control criteria for fault-free and second-fault scenario.

\begin{tabular}{lcccccc}
\hline \hline Fault-free situation & $\mathrm{E}(\mathrm{GJ})$ & Efficiency & $S\left(\mu \mathrm{rad}^{2} / 2\right)$ & GTV $(\mathrm{MNm})$ & $\operatorname{Max}\left(T_{g}\right)(\mathrm{kNm})$ & $\mathrm{STD}\left(T_{g}\right)(\mathrm{kNm})$ \\
\hline Proposed controller & 1.852 & $77 \%$ & 2123 & 42.74 & 30.52 & 12.28 \\
Reference controller & 1.785 & $74 \%$ & 20.95 & 0.5268 & 28.67 & 7.65 \\
Second-fault scenario & $\mathrm{E}(\mathrm{GJ})$ & Efficiency & $S\left(\mu \mathrm{rad}^{2} / 2\right)$ & GTV $(\mathrm{MNm})$ & $\operatorname{Max}\left(T_{g}\right)(\mathrm{kNm})$ & $\mathrm{STD}\left(T_{g}\right)(\mathrm{kNm})$ \\
\hline Proposed controller & \multirow{2}{*}{1.847} & $76 \%$ & 2992 & 42.53 & 35.30 & 12.49 \\
Reference controller & 1.374 & $57 \%$ & 264.4 & 75.44 & 35.30 & 11.23 \\
\hline \hline
\end{tabular}

fact, the adaptive structure of the controller and high variation of the neural networks output, which was used to estimate the unknown desired trajectory, i.e., Fig. 13, and also variation of $\hat{T}_{a}$ used in (27), led to this phenomenon. When considering Table II, high fluctuation of the generator torque has caused more $S$ on the drivetrain and GTV on the generator compared with the corresponding S and GTV for the ICGC. Nevertheless, when considering Figs. 10 and 12, it is obvious that the induced torsion angle and generator torque are in the same order as the ICGC results. So, it can be concluded that the proposed controller has similar performance in the fault-free case, as the ICGC, while both the efficiency and stress have been increased. The advantage of the proposed controller can be exploited in the faulty case when considering Tables II and V, in which the efficiency has been retained in the same order, while this number has been decreased approximately $13 \%$ for the ICGC. Also, the stress has been increased just 1.47 times more than the fault-free case, but this ratio is approximately 52.89 for the ICGC. Also, the actuator fault has been estimated, which can be used for maintenance purposes. The pitch actuator dynamic change, for nonzero desired pitch angle, $\beta_{d}$, was studied, and it was shown that the dynamic change can be accurately detected, as shown in Table III and Fig. 14. Also, the response of the pitch actuator under dynamic change was considered, and it was illustrated that the pitch angle has tracked the desired one, by using the proposed controller, faster than any other condition, either with or without dynamic change, as shown in Figs. 16 and 17. 
It should be clearly pointed out that, because of the negligible effect of the pitch actuator dynamic change in the partial load operation, where $\beta_{d}=0^{\circ}$, this case in not considered in Sec. VB 3, where just the effect of pitch bias has been studied. In fact, the proposed pitch controller is readily able to follow the $\beta_{d}$, whether fixed or variable, and detect dynamic change. Therefore, this controller is applicable in the full load region, where the $\beta_{d}$ can be simply tuned via a PI controller, ${ }^{2,7}$ which was not considered in this paper. In the selection of the controller parameters, a compromise should be taken between the boundedness of the controller behaviour and tracking performance. For example, because the RBFNN weight estimation, $\hat{\boldsymbol{\theta}}$, is the sliding surface function, a small $\sigma_{c}$ will cause large estimation weights. However, small $\sigma_{d_{1}}$ and $\sigma_{d_{2}}$ may make large adapting disturbance parameters and thus decrease the robustness to disturbances. In addition, large $k_{2, \omega_{\beta}}$ and $k_{2, \beta}$ may lead to saturated control input, i.e., pitch and generator torque. Actually, numerous offline response studies should be conducted to make the trade-off satisfactorily between the various contradictory objectives.

\section{CONCLUSIONS}

The optimum power point tracking of the nonlinear wind turbine, by using a nonlinear fault tolerant controller in the presence of actuator faults, was studied in this paper. The aim was to propose a new controller having functionality to fulfill the control objectives in both fault-free and faulty situations, satisfactorily. The wind speed and, accordingly, desired operational trajectory, at which wind turbines captures the most available energy were considered as unknown and so the desired trajectory was reconstructed by using a neural network scheme embedded into the proposed controller. By using the Lyapunov analysis, the boundedness of the closed-loop system behaviour with the proposed controller was guaranteed. The performance of the proposed controller was studied via numerical simulations in which the practical reference controller, i.e., constant gain controller, was used to evaluate the proposed controller results. The results showed that the proposed controller, contrary to the constant gain controller, was able to track the optimum power trajectory in both fault-free and faulty cases, with very close performance indices. In addition, the actuator faults were accurately estimated, which can be used for effective management of the required maintenance procedures.

${ }^{1}$ P. F. Odgaard and J. Stoustrup, “A benchmark evaluation of fault tolerant wind turbine control concepts," IEEE Trans. Control Syst. Technol. 23(3), 1221-1228 (2015).

${ }^{2}$ J. Lan, R. J. Patton, and X. Zhu, "Fault-tolerant wind turbine pitch control using adaptive sliding mode estimation," Renewable Energy 116, 219 (2018).

${ }^{3}$ P. Casau, P. Rosa, S. M. Tabatabaeipour, C. Silvestre, and J. Stoustrup, "A set-valued approach to FDI and FTC of wind turbines," IEEE Trans. Control Syst. Technol. 23(1), 245-263 (2015).

${ }^{4}$ R. Tiwari and N. R. Babu, "Recent developments of control strategies for wind energy conversion system," Renewable Sustainable Energy Rev. 66, 268-285 (2016).

${ }^{5}$ Z. Gao, C. Cecati, and S. X. Ding, "A survey of fault diagnosis and fault-tolerant techniques-Part I: Fault diagnosis with model-based and signal-based approaches," IEEE Trans. Ind. Electron. 62(6), 3757-3767 (2015).

${ }^{6}$ H. Habibi, H. Rahimi Nohooji, and I. Howard, "Power maximization of variable-speed variable-pitch wind turbines using passive adaptive neural fault tolerant control," Front. Mech. Eng. 12(3), 377-388 (2017).

${ }^{7}$ C. Sloth, T. Esbensen, and J. Stoustrup, "Robust and fault-tolerant linear parameter-varying control of wind turbines," Mechatronics 21(4), 645-659 (2011).

${ }^{8}$ H. Habibi, I. Howard, and R. Habibi, "Bayesian Sensor Fault Detection in a Markov Jump System," Asian J. Control 19(4), 1465-1481 (2017).

${ }^{9}$ P. F. Odgaard, J. Stoustrup, and M. Kinnaert, "Fault tolerant control of wind turbine-A benchmark model," in Fault Detection, Supervision and Safety of Technical Processes (2009), Vol. 42, pp. 155-160.

${ }^{10}$ F. D. Bianchi, H. De Battista, and R. J. Mantz, Wind Turbine Control Systems: Principles, Modelling and Gain Scheduling Design (Springer Science \& Business Media, 2006).

${ }^{11}$ F. Shi and R. Patton, "An active fault tolerant control approach to an offshore wind turbine model," Renewable Energy 75, 788-798 (2015).

${ }^{12}$ S. M. Tabatabaeipour, P. F. Odgaard, T. Bak, and J. Stoustrup, "Fault detection of wind turbines with uncertain parameters: A set-membership approach," Energies 5(7), 2424-2448 (2012).

${ }^{13} \mathrm{H}$. Sanchez, T. Escobet, V. Puig, and P. F. Odgaard, "Fault diagnosis of an advanced wind turbine benchmark using interval-based ARRs and observers," IEEE Trans. Ind. Electron. 62(6), 3783-3793 (2015).

${ }^{14} \mathrm{X}$. Wei, M. Verhaegen, and T. van Engelen, "Sensor fault detection and isolation for wind turbines based on subspace identification and Kalman filter techniques," Int. J. Adapt. Control 24(8), 687-707 (2010).

${ }^{15} \mathrm{X}$. Wei and M. Verhaegen, "Sensor and actuator fault diagnosis for wind turbine systems by using robust observer and filter," WiEn 14(4), 491-516 (2011). 
${ }^{16} \mathrm{H}$. Badihi, Y. Zhang, and H. Hong, "Fuzzy gain-scheduled active fault-tolerant control of a wind turbine," J. Franklin Inst. 351(7), 3677-3706 (2014).

${ }^{17}$ S. Sabzevari, A. Karimpour, M. Monfared, and M. B. Naghibi Sistani, "MPPT control of wind turbines by direct adaptive fuzzy-PI controller and using ANN-PSO wind speed estimator," J. Renewable Sustainable Energy 9(1), 013302 (2017).

${ }^{18} \mathrm{~S}$. Simani, "Overview of Modelling and Advanced Control Strategies for Wind Turbine Systems," Energies 8(12), 13395-13418 (2015).

${ }^{19}$ H. Habibi, A. Y. Koma, and A. Sharifian, "Power and velocity control of wind turbines by adaptive fuzzy controller during full load operation,” Iran. J. Fuzzy Syst. 13(3), 35-48 (2016), http://ijfs.usb.ac.ir/article_2428_0.html.

${ }^{20}$ H. Habibi, A. Y. Koma, and I. Howard, "Power Improvement of Non-Linear Wind Turbines during Partial Load Operation using Fuzzy Inference Control," Control Eng. Appl. Inf. 19(2), 31-42 (2017), http://www.ceai.srait.ro/ index.php?journal=ceai\&page $=$ article\&op=view\&path\%5B\%5D=4296.

${ }^{21}$ Y. Song, Z. Zhang, P. Li, W. Wang, and M. Qin, "Robust adaptive variable speed control of wind power systems without wind speed measurement," J. Renewable Sustainable Energy 5(6), 063115 (2013).

${ }^{22}$ B. Boukhezzar and H. Siguerdidjane, "Nonlinear control of a variable-speed wind turbine using a two-mass model," IEEE Trans. Energy Convers. 26(1), 149-162 (2011).

${ }^{23}$ K. Z. Østergaard, P. Brath, and J. Stoustrup, "Estimation of effective wind speed," J. Phys.: Conf. Ser. 75, 012082 (2007).

${ }^{24} \mathrm{H}$. Jafarnejadsani, J. Pieper, and J. Ehlers, "Adaptive control of a variable-speed variable-pitch wind turbine using radialbasis function neural network,” IEEE Trans. Control Syst. Technol. 21(6), 2264-2272 (2013).

${ }^{25}$ P. F. Odgaard, J. Stoustrup, and M. Kinnaert, "Fault-tolerant control of wind turbines: A benchmark model," IEEE Trans. Control Syst. Technol. 21(4), 1168-1182 (2013).

${ }^{26}$ S. Heier, Grid Integration of Wind Energy Conversion Systems (Wiley, 1998).

${ }^{27} \mathrm{H}$. Badihi, Y. Zhang, and H. Hong, "Wind turbine fault diagnosis and fault-tolerant torque load control against actuator faults," IEEE Trans. Control Syst. Technol. 23(4), 1351-1372 (2015).

${ }^{28}$ D. Liu, J. Guo, Y. Huang, and W. Wang, "An active power control strategy for wind farm based on predictions of wind turbine's maximum generation capacity,” J. Renewable Sustainable Energy 5(1), 013121 (2013).

${ }^{29}$ S. S. Ge and C. Wang, "Adaptive neural control of uncertain MIMO nonlinear systems," IEEE Trans. Neural Network Learn. Syst. 15(3), 674-692 (2004).

${ }^{30}$ Y. Song and J. Guo, "Neuro-Adaptive Fault-Tolerant Tracking Control of Lagrange Systems Pursuing Targets With Unknown Trajectory," IEEE Trans. Ind. Electron. 64(5), 3913-3920 (2017).

${ }^{31}$ B. Ren, S. S. Ge, K. P. Tee, and T. H. Lee, "Adaptive neural control for output feedback nonlinear systems using a barrier Lyapunov function," IEEE T. Neural Network 21(8), 1339-1345 (2010).

${ }^{32} \mathrm{H}$. Schulte and E. Gauterin, "Fault-tolerant control of wind turbines with hydrostatic transmission using Takagi-Sugeno and sliding mode techniques," Annu. Rev. Control 40, 82-92 (2015). 